\title{
Design, Dynamics, and Workspace of a Hybrid-Driven-Based Cable Parallel Manipulator
}

\author{
Bin Zi, ${ }^{1,2}$ Jianbin Cao, ${ }^{1}$ Zhencai Zhu, ${ }^{1}$ and Peter Mitrouchev ${ }^{3}$ \\ ${ }^{1}$ School of Mechanical and Electrical Engineering, China University of Mining and Technology, Xuzhou, Jiangsu 221116, China \\ ${ }^{2}$ Chair of Mechanics and Robotics, University of Duisburg-Essen, 47057 Duisburg, Germany \\ ${ }^{3}$ G-SCOP Laboratory, Grenoble-INP/UJF-Grenoble 1/CNRS, 46 Avenue Félix Viallet, 38031 Grenoble Cedex 1, France
}

Correspondence should be addressed to Bin Zi; binzi.cumt@163.com

Received 8 September 2012; Revised 9 December 2012; Accepted 27 December 2012

Academic Editor: G. Rega

Copyright (C) 2013 Bin Zi et al. This is an open access article distributed under the Creative Commons Attribution License, which permits unrestricted use, distribution, and reproduction in any medium, provided the original work is properly cited.

\begin{abstract}
The design, dynamics, and workspace of a hybrid-driven-based cable parallel manipulator (HDCPM) are presented. The HDCPM is able to perform high efficiency, heavy load, and high-performance motion due to the advantages of both the cable parallel manipulator and the hybrid-driven planar five-bar mechanism. The design is performed according to theories of mechanism structure synthesis for cable parallel manipulators. The dynamic formulation of the HDCPM is established on the basis of NewtonEuler method. The workspace of the manipulator is analyzed additionally. As an example, a completely restrained HDCPM with 3 degrees of freedom is studied in simulation in order to verify the validity of the proposed design, workspace, and dynamic analysis. The simulation results, compared with the theoretical analysis, and the case study previously performed show that the manipulator design is reasonable and the mathematical models are correct, which provides the theoretical basis for future physical prototype and control system design.
\end{abstract}

\section{Introduction}

In manipulation systems, it is always a goal to continuously improve the range of motion, accuracy, and load capacities. Compared with the traditional mechanical structures, parallel mechanisms have the advantages of high dexterity and accuracy. Cable parallel manipulators (CPMs) are categorized as a type of parallel manipulators. They are a variant of the Gough-Stewart platform in which rigid extensible legs are replaced by cables stored spools. CPMs have several advantages over rigid-link mechanisms, such as (i) remote location of motors and controls, (ii) rapid deployability, (iii) potentially large workspaces and (iv) high load capacity; (v) reliability $[1,2]$. For the preceding characteristics, the CPMs have received a lot of attention since 1980s [3]. These manipulators, referred to as the overhead crane and rotary crane, are useful for many applications in hazardous environments in order to enable the manipulation of heavy objects $[4,5]$.

The first written information on use of hoist mechanism in ancient Greece appeared around 530 BC, which is concerned with the construction of the first temple of Artemis in Ephesus, and temples at Selinous, temples of Apollo at Syracuse and at Corinth. After 515 BC, cranes were in common use $[6,7]$. The CPMs consist of a fixed base and a centrally located end-effector, attached to moving payload, connected by cables whose tension is maintained along the tracked trajectory $[8,9]$. However, due to the unilateral driving property of the cables, maintaining positive cable tension is essential to maneuver the end-effector. As a result, the number of driving cables must be more than the number of degrees of freedom (DOF) of the end-effector in order to obtain a completely restrained manipulation [10]. This type of technology is now well known, and several studies of the CPMs have been undertaken. For instance, Gosselin and Barrette [11] presented a fundamental and systematic analysis of a planar CPM. Design and performances of a 4-cable driven parallel manipulator have been presented by Ottaviano et al. [12]. Flatness-based trajectory tracking control for a three-cable suspension manipulator has been investigated by Heyden and Woernle [13]. Due to cable slippage on the drum, Baser and Konukseven developed an 


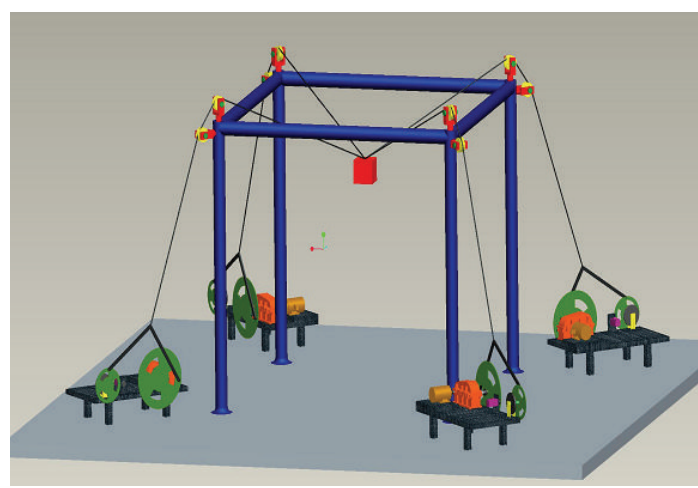

FIgURE 1: Three-dimensional model of the HDCPM.

analytical method for predicting the transmission error [14]. Different aspects like kinematics [15], workspace characteristics [16], dimensional optimization [17], and statics [18] were extensively investigated.

As we are aware, there are relatively fewer published works on the drive system of the CPMs, which is the main contribution of this work. The existing research on the drive system of the CPMs usually uses the low power controllable motor. In the recent years, as the CPMs have been investigated widely for various applications in modern manufacturing, they are required not only for operations with high accuracy and high payload, but also for output with greater flexibility, which can change the law of output motion quickly and conveniently [19]. Nevertheless, the low power controllable motor cannot directly drive the high-loading CPM due to the restrictions of power and torque. Hence, it is necessary to carry out for a new-type driven system for the CPMs.

The hybrid-driven planar five-bar mechanism (HDPM) is a kind of machine whose drive system consists of a constant velocity $(\mathrm{CV})$ motor and a servomotor [20]. The CV motor provides main power and motion required; however, it lacks high controllability. On the other hand, the servomotor acts as a motion regulation device which suffers from the limited power capacity. Therefore, the HDPM can take the advantage of the complementary characteristics of both motors to generate a programmable range of highly nonlinear output motions with high power capacities [21]. In fact, the HDPM can be seen as a type of servomechanism [22]. In this investigation the design of a hybrid-driven-based cable parallel manipulator (HDCPM) which combines four groups of HDPMs with a 4-cable parallel manipulator is presented. The HDCPM can take the advantages of the characteristics of both mechanisms to generate a high-performance output motion with high efficiency and heavy payload [23].

It is well known that dynamic performance has a great impact on the operation of the manipulator, and this is on the basis of its dynamic control. As demonstrated in [24], servomechanism dynamics constitute an important component of the complete robot dynamics. Therefore, for the HDCPM, the dynamics should not only consider the CPM, but also include the HDPM (servomechanism). However, the literature on the dynamics of the CPM system including the linkage (servomechanism) dynamics is sparse. Aiming at the HDCPM, this paper is intended to study the completely dynamic modeling including the HDPM actuator dynamics on the basis of Newton-Euler method. The latter is used to solve the inverse kinematic and dynamic problems of the HDCPM on condition that the operation path of the endeffector has been planned.

The workspace is also one of the most useful measures for the evaluation of the manipulator performances [25-27]. Pusey et al. [28] defined the workspace of a CPM as the set of points where the centre of gravity of the end-effector can be reached with admissible tensions in all cables. Apart from the tension condition, workspace of the HDCPM is also limited by geometrical conditions, which include the workspace of the HDPM actuator, length of cables, and motion range of end-effector for the HDCPM. This paper discusses the process of workspace formation of the HDCPM in detail.

In the rest of the paper, Section 2 describes the design model of the HDCPM. In the following, dynamic modeling of the HDCPM is performed based on Newton-Euler method in Section 3. Workspace analysis of the HDCPM is provided in Section 4. In Section 5, illustrative simulation studies highlight its performances. Finally, some concluding remarks and future studies are summarized in Section 6.

\section{Mechanism Description}

In this paper, for the purpose of analytical modeling and numerical analysis, the three-dimensional design model of the completely restrained HDCPM with three translational motions is taken as an example (see Figure 1). The HDCPM suspends an end-effector by four cables and restrains all motion degrees of freedom for the object using the cable force when the end-effector moves within the workspace. For each cable, one end is connected to the end-effector, the other one rolls through a pulley fixed on the top of the relative cable tower rack and then is fed into the HDPM. The HDCPM comprises of two modules: (1) the CPM consisting of four-cable tower racks, four cables, pulley struts, pulleys, girder, and cargo (i.e., end-effector); (2) four groups of HDPMs containing three-phase asynchronous motors, servomotors, reducers, and double crank five-bar linkage; the asynchronous motors are connected using the pulley transmission mechanisms, while the servomotors and the reducers are linked by couplings. At the same time, the pulley transmission mechanisms and reducers are joined to the double crank planar five-bar linkage. The double crank planar five-bar linkage is made up of a large crank disk, a long connecting rod, a small crank disk, and a short connecting rod. Both long and short connecting rods are articulated with each other and attached to the cable. Moreover, each tower rack of the CPM is equipped with a cable guide pulley. The mechanical configuration of the HDCPM is illustrated in Figure 2.

The working process of the HDCPM is as follows. The $\mathrm{CV}$ three-phase asynchronous motors and servomotors are power sources. On the one hand, the servomotor and the reducer are linked by the coupling, then the servomotors through a small crank disk are connected with a short rod 


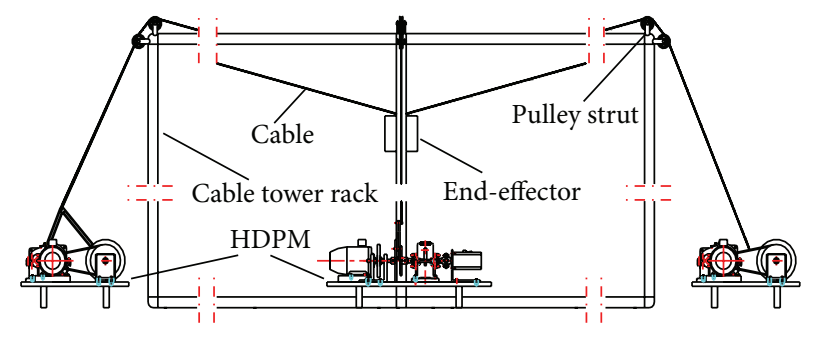

(a)

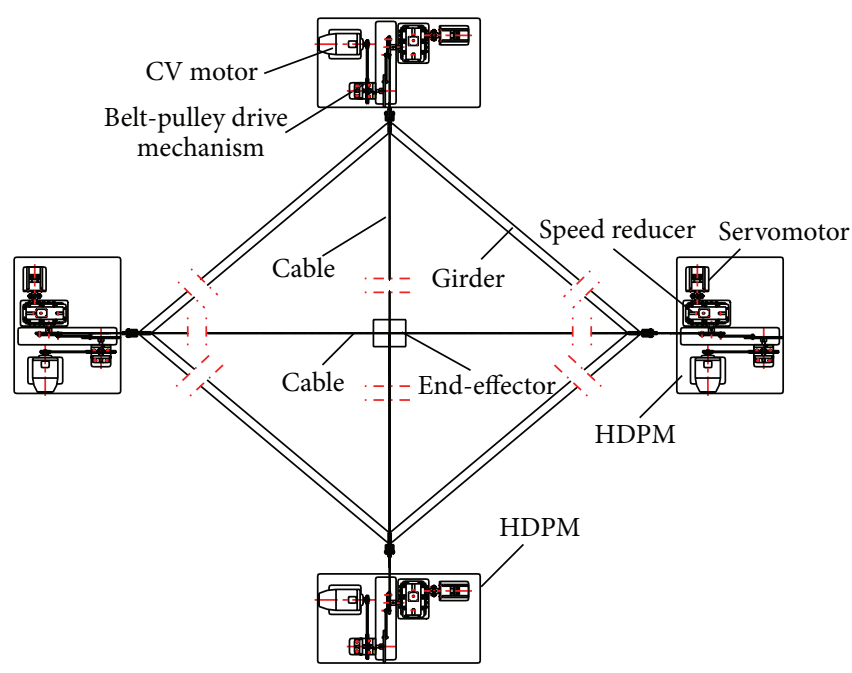

(b)

Figure 2: Mechanical configuration of the HDCPM: (a) front view, (b) top view.

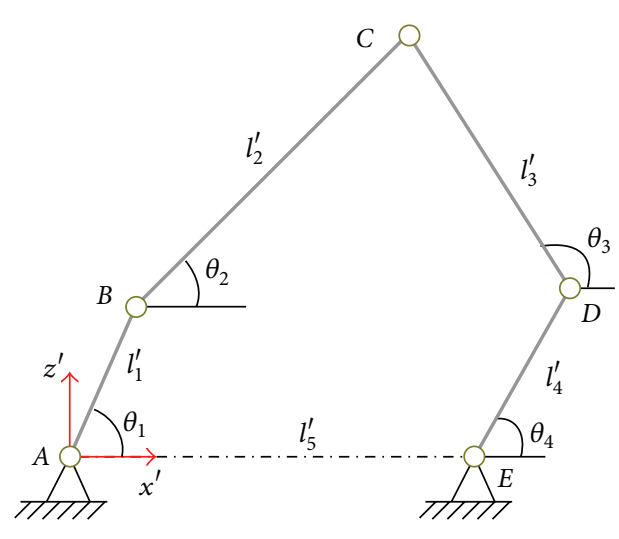

(a)

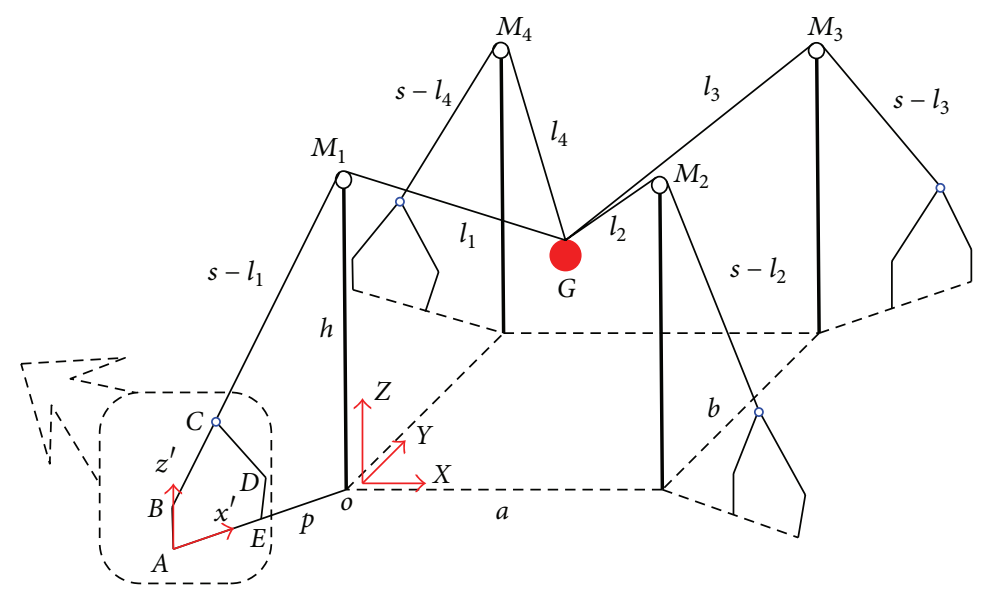

(b)

FIGURE 3: Structure model (a) of the HDPM and schematic sketch (b) of the HDCPM.

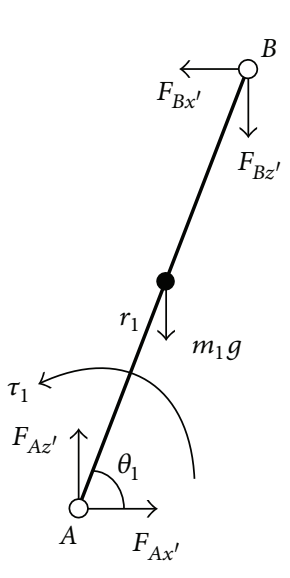

(a)

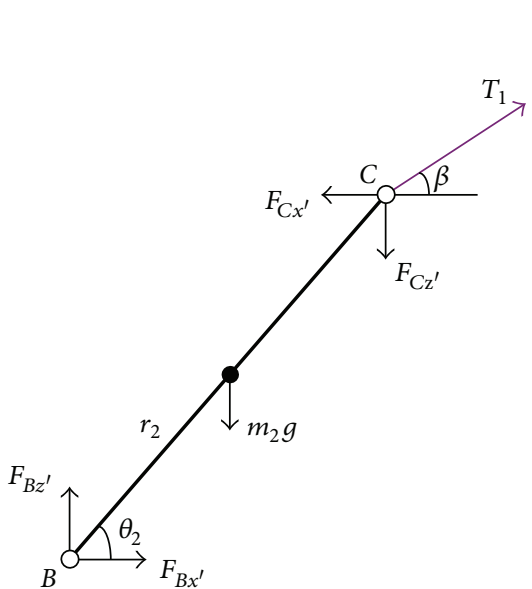

(b)
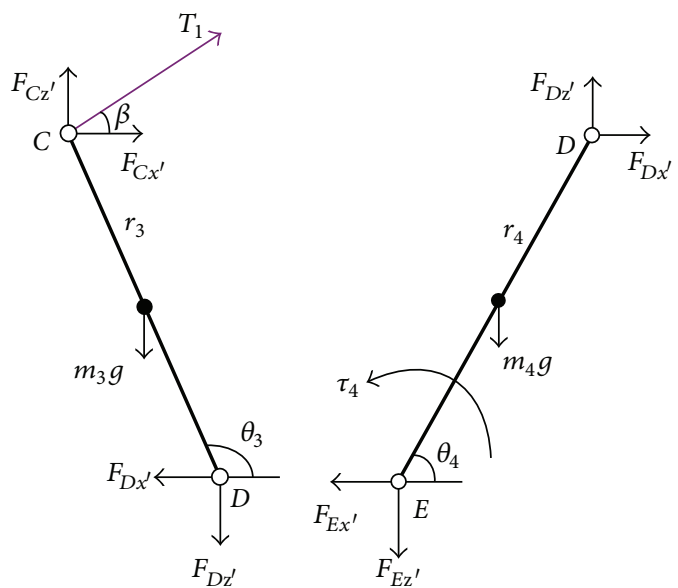

(c) (d)

FIgURE 4: Free body diagrams of the HDPM: (a) link $A B$, (b) link $B C$, (c) link $C D$, and (d) link $E D$. 


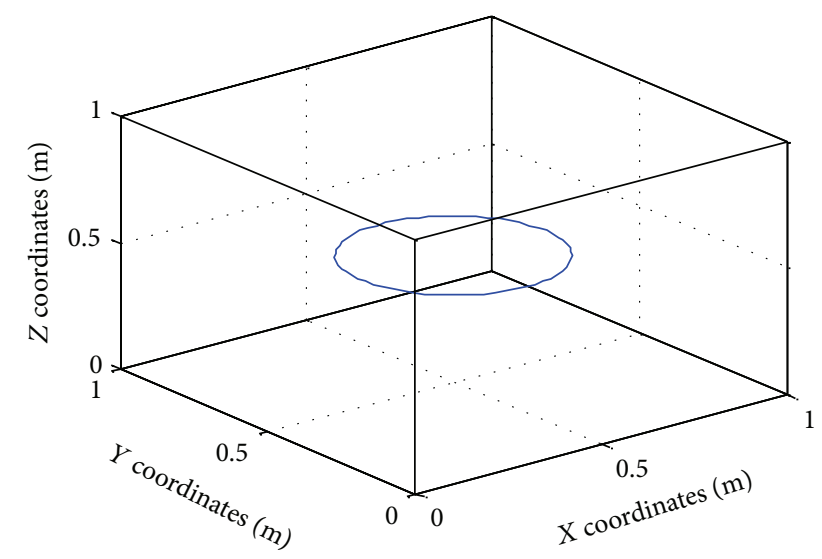

FIGURE 5: Following trajectory of the circle motion.

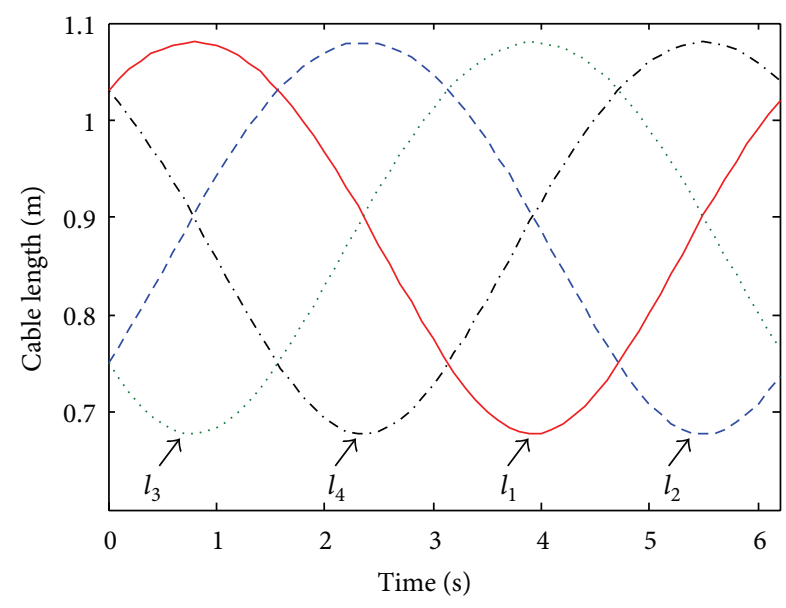

FIgURE 6: Time behavior of cable length for Case 1.

connection thus adjusting the output motion of the HDPM. On the other hand, each CV three-phase asynchronous motor is connected with the pulley transmission mechanisms, then it links to a long connecting rod by a large crank disk, thus providing the main power for the HDCPM. These two types of input motions are hinged through the short and long connecting rod, so that the power distribution and other characteristics of the HDPM are improved while ensuring the output motions. Four groups of the HDPM with the same structure produce rotary motion, thus the four cables can be driven in order to realize the output motion trajectory of the end-effector.

\section{Dynamic Modeling}

A simple schematic sketch of the HDCPM structure model with the associated coordinate systems is depicted in Figure 3. At the bottom of one cable tower rack a global coordinate system $O(X Y Z)$ is established. The end-effector has location coordinates $G(x, y, z)$. The distance between each cable tower rack top $M_{i}\left(x_{i}, y_{i}, z_{i}\right)$ and the end-effector is $l_{i}(i=1,2,3,4)$. The four cable tower racks have same heighth and are arrayed in a rectangle on the ground, where deformation is ignored.

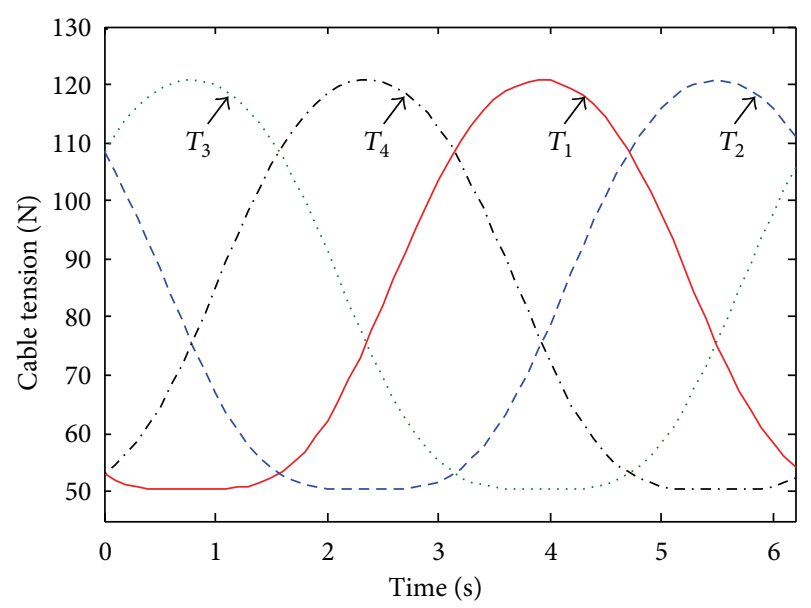

Figure 7: Curves of cable tension for Case 1.

In order to simplify the model, the cables are treated as a massless body with no deformation, with length $s$. The bottom of the HDPM corresponds to the joint $A$ as the origin, where a local coordinate system $A\left(x^{\prime} y^{\prime} z^{\prime}\right)$ is established.

Link $A B$ driven by a $C V$ motor and link $E D$ driven by a servomotor are the two driving links (Figure 2(a)), the joint $C$ is the output. There are two independent constraints in the HDPM, which can be used to derive the kinematic equation of the HDPM:

$$
\begin{gathered}
l_{1}^{\prime} \cos \theta_{1}+l_{2}^{\prime} \cos \theta_{2}-l_{3}^{\prime} \cos \theta_{3}-l_{4}^{\prime} \cos \theta_{4}-l_{5}^{\prime}=0, \\
l_{1}^{\prime} \sin \theta_{1}+l_{2}^{\prime} \sin \theta_{2}-l_{3}^{\prime} \sin \theta_{3}-l_{4}^{\prime} \sin \theta_{4}=0 .
\end{gathered}
$$

In (1) $\theta_{1}$ and $\theta_{4}$ are known. Consequently $\theta_{2}$ and $\theta_{3}$ can be calculated as

$$
\begin{gathered}
\theta_{3}\left(\theta_{1}, \theta_{4}\right)=\arccos \left(\frac{C}{\sqrt{A^{2}+B^{2}}}\right)+\arctan \left(\frac{\mu}{\lambda}\right), \\
\theta_{2}\left(\theta_{1}, \theta_{4}\right)=\arctan \left(\frac{\mu+l_{3}^{\prime} \sin \theta_{3}}{\lambda+l_{3}^{\prime} \cos \theta_{3}}\right),
\end{gathered}
$$

where $A=2 l_{3}^{\prime} \lambda, B=2 l_{3}^{\prime} \mu, C=l_{2}^{\prime 2}-l_{3}^{\prime 2}-\lambda^{2}-\mu^{2}, \lambda=l_{4}^{\prime} \cos \theta_{4}-$ $l_{1}^{\prime} \cos \theta_{1}+l_{5}^{\prime}, \mu=l_{4}^{\prime} \sin \theta_{4}-l_{1}^{\prime} \sin \theta_{1}$.

Let

$$
\begin{gathered}
x_{C}^{\prime}=l_{1}^{\prime} \cos \theta_{1}+l_{2}^{\prime} \cos \theta_{2}=l_{5}^{\prime}+l_{3}^{\prime} \cos \theta_{3}+l_{4}^{\prime} \cos \theta_{4}, \\
z_{C}^{\prime}=l_{1}^{\prime} \sin \theta_{1}+l_{2}^{\prime} \sin \theta_{2}=l_{3}^{\prime} \sin \theta_{3}+l_{4}^{\prime} \sin \theta_{4}
\end{gathered}
$$

be the coordinates of joint $C$ in the local coordinate system. According to the geometric relationship of the HDCPM, $l_{1}$ 


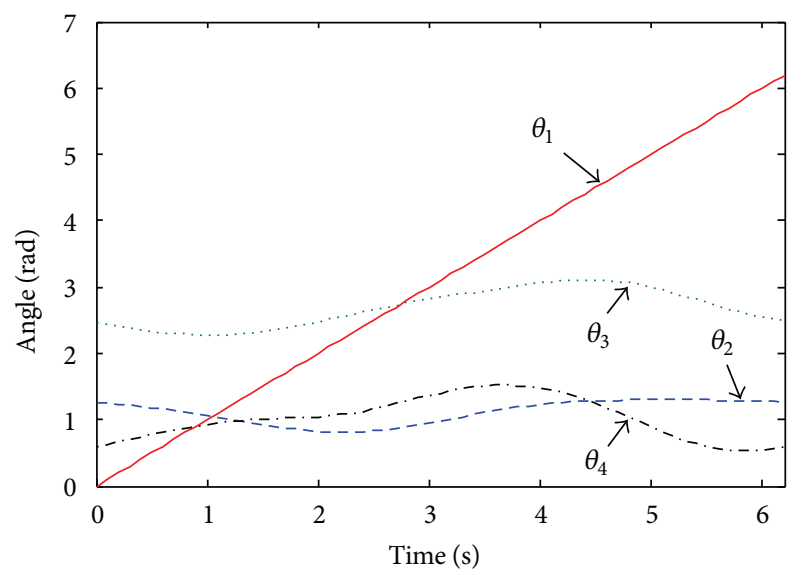

(a)

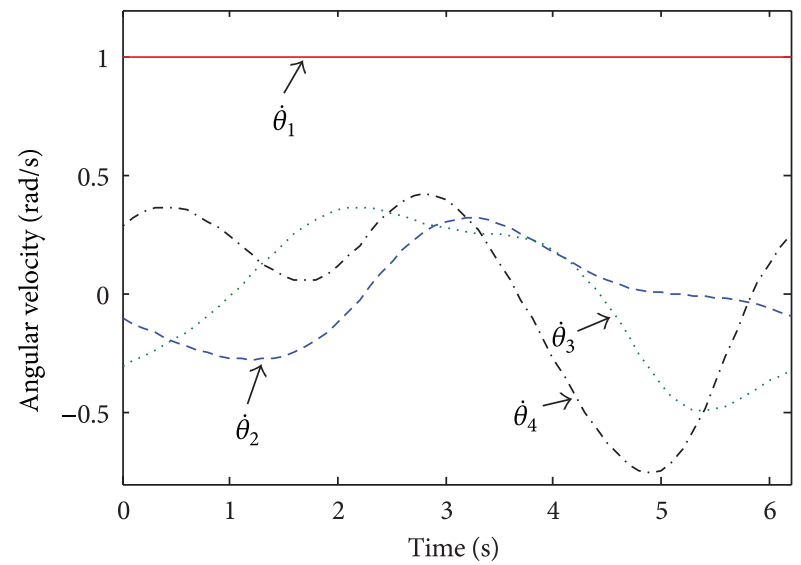

(b)

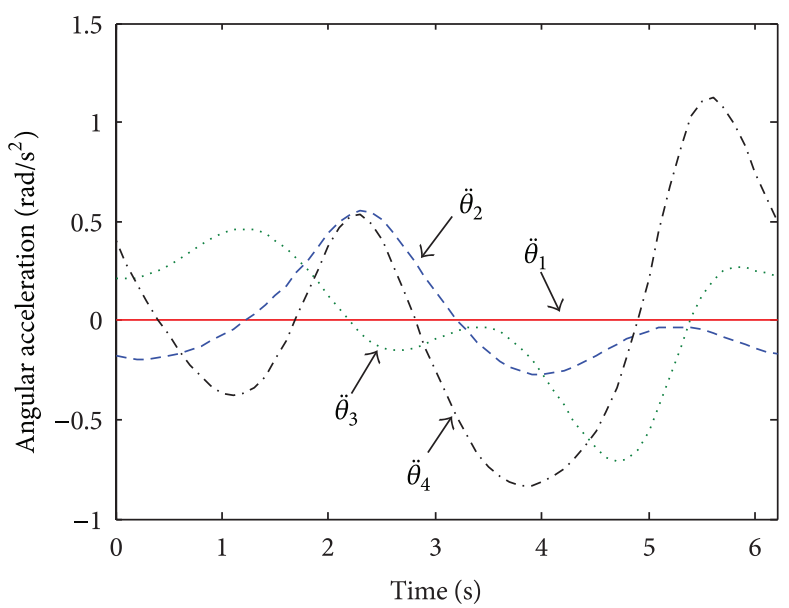

(c)

Figure 8: Curves of the links motion parameters of the HDPM for Case 1: (a) angle, (b) angular velocity, and (c) angular acceleration.

$$
\begin{gathered}
x_{\min }^{\prime}=-\left(l_{1}^{\prime}+l_{2}^{\prime}\right), x_{\max }^{\prime}=l_{3}^{\prime}+l_{4}^{\prime}+l_{5}^{\prime} \\
z_{\min }^{\prime}=-\max \left\{l_{1}^{\prime}+l_{2}^{\prime}, l_{3}^{\prime}+l_{4}^{\prime}\right\}, z_{\max }^{\prime}=\max \left\{l_{1}^{\prime}+l_{2}^{\prime}, l_{3}^{\prime}+l_{4}^{\prime}\right\} \\
x^{\prime}=x_{\min }^{\prime}, z^{\prime}=z_{\min }^{\prime}
\end{gathered}
$$

Do While $z^{\prime} \leq z_{\max }^{\prime}$

Do While $x^{\prime} \leq x_{\max }^{\prime}$

If $\left|l_{1}^{\prime}-l_{2}^{\prime}\right|<L_{A C}<l_{1}^{\prime}+l_{2}^{\prime}$ and $\left|l_{3}^{\prime}-l_{4}^{\prime}\right|<L_{C E}<l_{3}^{\prime}+l_{4}^{\prime}$ Then

From $l_{1}=s-\sqrt{\left(p+l_{5}^{\prime}-x_{C}^{\prime}\right)^{2}+\left(h-z_{C}^{\prime}\right)^{2}}, l_{1}$ is determined.

Similarly, $l_{2}, l_{3}$, and $l_{4}$ can be calculated, respectively.

Record $l_{i}$

\section{End If}

$$
x^{\prime}=x^{\prime}+\Delta x^{\prime}
$$

\section{Loop}

$$
z^{\prime}=z^{\prime}+\Delta z^{\prime}
$$

\section{Loop}

Various combinations of $l_{i}$ for calculating coordinates $(x, y, z)$ by

$x=\left(l_{1}^{2}-l_{2}^{2}+a^{2}\right) / 2 a, y=\left(l_{1}^{2}-l_{4}^{2}+b^{2}\right) / 2 b, z=h-\sqrt{l_{1}^{2}-x^{2}-y^{2}}$

and those satisfying motion range of the end-effector $(0<x<a, 0<y<b, 0<z<h)$ and tension condition $\left(T_{i}>0\right)$ will generate the workspace 


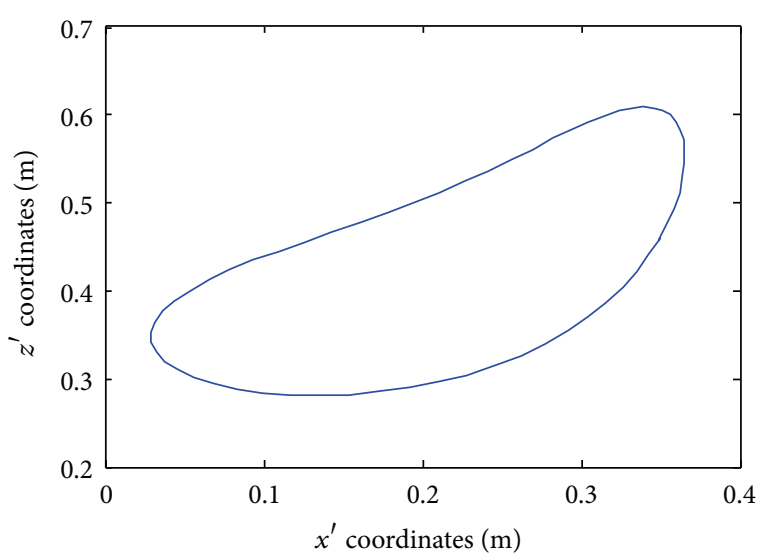

Figure 9: Trajectory of joint $C$ in $x^{\prime}-z^{\prime}$ plane for Case 1.

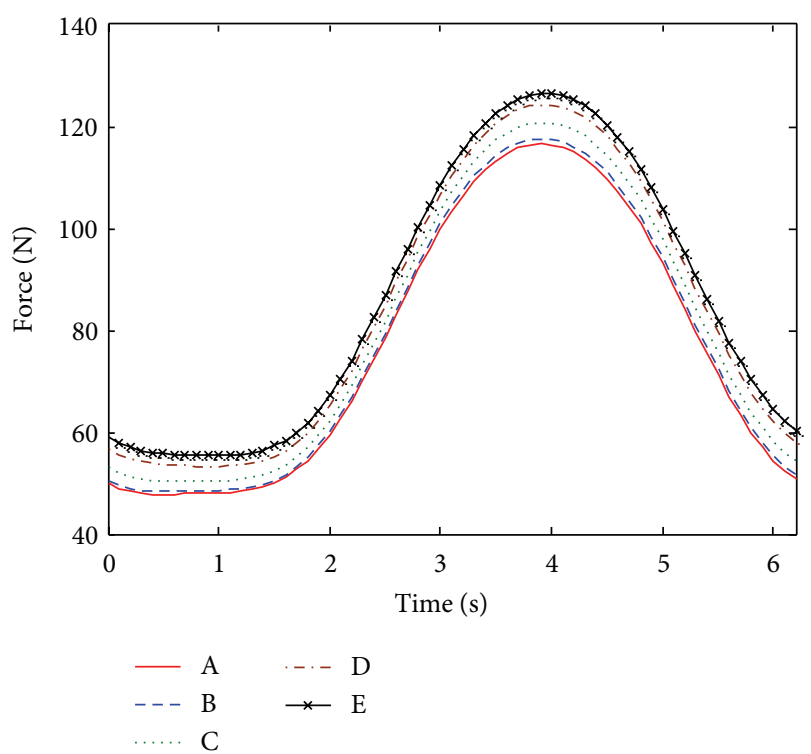

FIGURE 10: Resultant force curves of the joints of the HDPM for Case 1.

can be derived as

$$
l_{1}=S-\sqrt{\left(p+l_{5}^{\prime}-x_{C}^{\prime}\right)^{2}+\left(h-z_{C}^{\prime}\right)^{2}}
$$

where $p$ represents the distance $\overline{E O}$ and $s$ is the total length of cable. Similarly, $l_{2}, l_{3}$, and $l_{4}$ can be derived, respectively.

The relationships between the cable length $l_{i}$ and the endeffector location $G(x, y, z)$ can be easily obtained as follows:

$$
\begin{gathered}
l_{1}=\sqrt{x^{2}+y^{2}+(z-h)^{2}}, \\
l_{2}=\sqrt{(x-a)^{2}+y^{2}+(z-h)^{2}}, \\
l_{3}=\sqrt{(x-a)^{2}+(y-b)^{2}+(z-h)^{2}}, \\
l_{4}=\sqrt{x^{2}+(y-b)^{2}+(z-h)^{2}}
\end{gathered}
$$

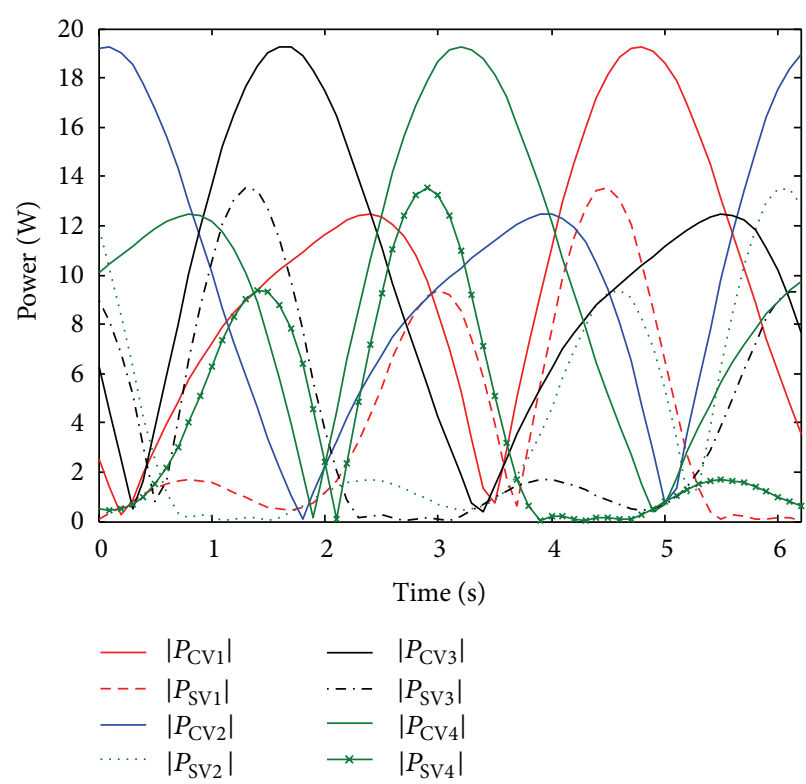

FIGURE 11: Motor absolute value power curves of the four groups of HDPMs of the HDCPM.

resulting in

$$
\begin{gathered}
x=\frac{l_{1}^{2}-l_{2}^{2}+a^{2}}{2 a}, \\
y=\frac{l_{1}^{2}-l_{4}^{2}+b^{2}}{2 b}, \\
z=h-\sqrt{l_{1}^{2}-x^{2}-y^{2}} .
\end{gathered}
$$
in

Newton's law for the end-effector of the HDCPM results

$$
m\left[\begin{array}{c}
\ddot{x} \\
\ddot{y} \\
\ddot{z}
\end{array}\right]=\sum_{i=1}^{4} \vec{T}_{i}+\left[\begin{array}{c}
0 \\
0 \\
-m g
\end{array}\right],
$$

where $T_{i}(i=1,2,3,4)$ is a tension cable force, that is, the driving force of cable $i$ exerted on the end-effector for the HDCPM, and $\vec{T}_{i}$ is force vector.

Thus the dynamic model can be expressed as follows:

$$
N_{3 \times 4} T_{4 \times 1}=M_{3 \times 1}
$$

where

$$
N_{3 \times 4}=\left[\begin{array}{cccc}
\frac{-x}{l_{1}} & \frac{a-x}{l_{2}} & \frac{a-x}{l_{3}} & \frac{-x}{l_{4}} \\
\frac{-y}{l_{1}} & \frac{-y}{l_{2}} & \frac{b-y}{l_{3}} & \frac{b-y}{l_{4}} \\
\frac{h-z}{l_{1}} & \frac{h-z}{l_{2}} & \frac{h-z}{l_{3}} & \frac{h-z}{l_{4}}
\end{array}\right]
$$




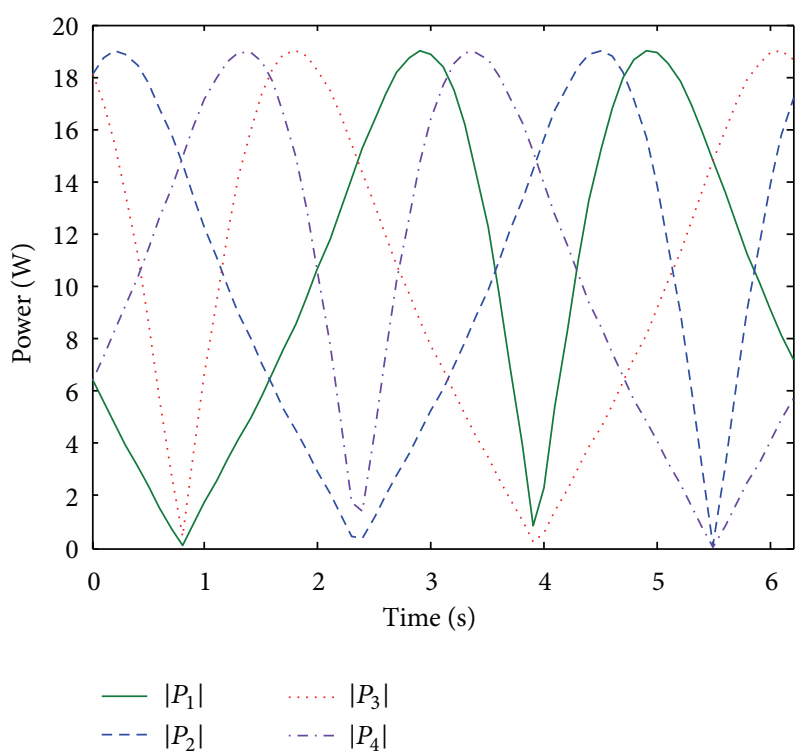

FIGURE 12: Servomotor absolute value power curves of the conventional CPM.

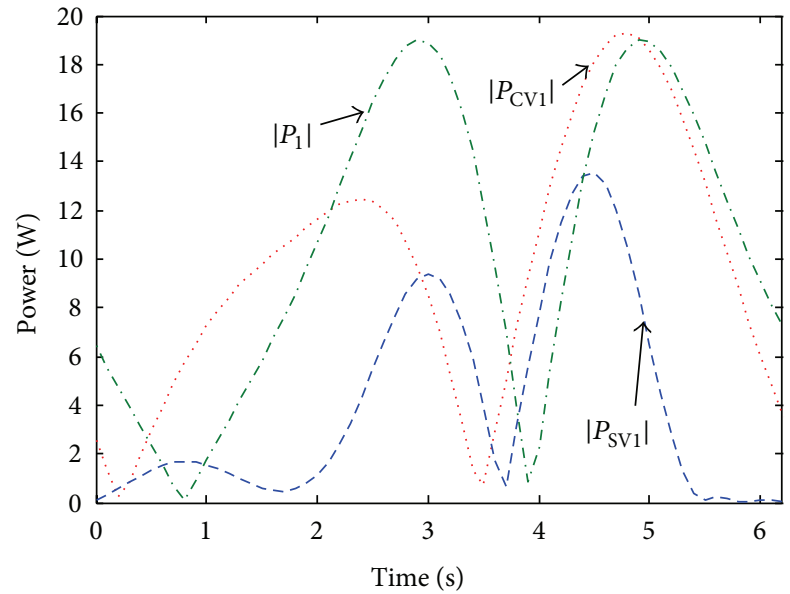

FIGURE 13: Comparison of absolute value power curves between the HDCPM and conventional CPM.

is the matrix of coordinate transformer, $T_{4 \times 1}=$ $\left[\begin{array}{llll}T_{1} & T_{2} & T_{3} & T_{4}\end{array}\right]^{T}$ is the vector of cable tension, $M_{3 \times 1}=$ $\left[\begin{array}{lll}m \ddot{x} & m \ddot{y} & m g+m \ddot{z}\end{array}\right]^{T}$ is the vector of inertia force, $m$ is mass of the end-effector, and $g$ the gravitational acceleration.

The dynamic model of the HDCPM is presented in two parts. The first is directed to the structural model (CPM) above, the other one to the actuator dynamics (servomechanism). Applying the Newton-Euler equation establishes the dynamic model of the HDPM. The force analysis of each link of the HDPM is shown in Figure 4. $\tau_{1}$ and $\tau_{4}$ represent the driving torque of the $\mathrm{CV}$ motor on link $A B$ and the servomotor on link $E D$, respectively; $\beta=\arctan ((h-$ $\left.\left.l_{1}^{\prime} \sin \theta_{1}-l_{2}^{\prime} \sin \theta_{2}\right) /\left(p+l_{5}^{\prime}-l_{1}^{\prime} \cos \theta_{1}-l_{2}^{\prime} \cos \theta_{2}\right)\right)$ is the angle between cable tension and $x^{\prime}$-coordinate.
By the force analysis mentioned previously, the NewtonEuler formulation of the links can be listed as follows.

Link $A B$ :

$$
\begin{aligned}
& F_{A x^{\prime}}-F_{B x^{\prime}}=m_{1} \ddot{x}_{1}^{\prime}, \\
& F_{A z^{\prime}}-m_{1} g-F_{B z^{\prime}}=m_{1} \ddot{z}_{1}^{\prime}, \\
& \tau_{1}+F_{A x^{\prime}} r_{1} \sin \theta_{1}-F_{A z^{\prime}} r_{1} \cos \theta_{1}+F_{B x^{\prime}}\left(l_{1}^{\prime}-r_{1}\right) \sin \theta_{1} \\
& \quad \quad-F_{B z^{\prime}}\left(l_{1}^{\prime}-r_{1}\right) \cos \theta_{1}=J_{1} \ddot{\theta}_{1} .
\end{aligned}
$$

\section{Link $B C$ :}

$$
\begin{aligned}
& T_{1} \cos \beta+F_{B x^{\prime}}-F_{C x^{\prime}}=m_{2} \ddot{x}_{2}^{\prime}, \\
& T_{1} \sin \beta+F_{B z^{\prime}}-F_{C z^{\prime}}-m_{2} g=m_{2} \ddot{z}_{2}^{\prime}, \\
& F_{B x^{\prime}} r_{2} \sin \theta_{2}-F_{B z^{\prime}} r_{2} \cos \theta_{2} \\
& \quad+\left(F_{C x^{\prime}}-T_{1} \cos \beta\right)\left(l_{2}^{\prime}-r_{2}\right) \sin \theta_{2} \\
& \quad+\left(T_{1} \sin \beta-F_{C z^{\prime}}\right)\left(l_{2}^{\prime}-r_{2}\right) \cos \theta_{2}=J_{2} \ddot{\theta}_{2} .
\end{aligned}
$$

Link $C D$ :

$$
\begin{aligned}
& T_{1} \cos \beta+F_{C x^{\prime}}-F_{D x^{\prime}}=m_{3} \ddot{x}_{3}^{\prime}, \\
& T_{1} \sin \beta+F_{C z^{\prime}}-m_{3} g-F_{D z^{\prime}}=m_{3} \ddot{z}_{3}^{\prime}, \\
& \left(F_{C z \prime}+T_{1} \sin \beta\right) r_{3} \cos \theta_{3} \\
& \quad-\left(F_{C x^{\prime}}+T_{1} \cos \beta\right) r_{3} \sin \theta_{3}-F_{D x^{\prime}}\left(l_{3}^{\prime}-r_{3}\right) \sin \theta_{3} \\
& \quad+F_{D z^{\prime}}\left(l_{3}^{\prime}-r_{3}\right) \cos \theta_{3}=J_{3} \ddot{\theta}_{3} .
\end{aligned}
$$

Link $E D$ :

$$
\begin{aligned}
& F_{D x^{\prime}}-F_{E x^{\prime}}=m_{4} \ddot{x}_{4}^{\prime}, \\
& F_{D z^{\prime}}-m_{4} g-F_{E z^{\prime}}=m_{4} \ddot{z}_{4}^{\prime}, \\
& \tau_{4}-F_{D x^{\prime}} r_{4} \sin \theta_{4}+F_{D z^{\prime}} r_{4} \cos \theta_{4} \\
& \quad-F_{E x^{\prime}}\left(l_{4}^{\prime}-r_{4}\right) \sin \theta_{4} \\
& \quad+F_{E z^{\prime}}\left(l_{4}^{\prime}-r_{4}\right) \cos \theta_{4}=J_{4} \ddot{\theta}_{4},
\end{aligned}
$$

where $m_{j}, l_{j}^{\prime}, J_{j}, r_{j},\left(x_{j}^{\prime}, z_{j}^{\prime}\right)(j=1,2,3,4)$, represent the mass, length, inertia, distance between the mass centre and the joint, and coordinates of the mass centre of each link, respectively.

The equation can be summarized as

$$
A_{12 \times 14} H_{14 \times 1}=B_{12 \times 1},
$$


where

$A_{12 \times 14}$

\begin{tabular}{|c|c|c|c|c|c|c|c|c|c|c|c|c|}
\hline 1 & 0 & -1 & 0 & 0 & 0 & 0 & 0 & 0 & 0 & 0 & 0 & 0 \\
\hline 0 & 1 & 0 & -1 & 0 & 0 & 0 & 0 & 0 & 0 & 0 & 0 & 0 \\
\hline$r_{1} s_{1}$ & $-r_{1} c_{1}$ & $\left(l_{1}^{\prime}-r_{1}\right) s_{1}$ & $\left(r_{1}-l_{1}^{\prime}\right) c_{1}$ & 0 & 0 & 0 & 0 & 0 & 0 & 0 & 0 & 1 \\
\hline 0 & 0 & 1 & 0 & -1 & 0 & 1 & 0 & 0 & 0 & 0 & 0 & 0 \\
\hline 0 & 0 & 0 & 1 & 0 & -1 & 0 & 1 & 0 & 0 & 0 & 0 & 0 \\
\hline 0 & 0 & $r_{2} s_{2}$ & $-r_{2} c_{2}$ & $\left(l_{2}^{\prime}-r_{2}\right) s_{2}$ & $\left(r_{2}-l_{2}^{\prime}\right) c_{2}$ & $\left(r_{2}-l_{2}^{\prime}\right) s_{2}$ & $\left(l_{2}^{\prime}-r_{2}\right) c_{2}$ & 0 & 0 & 0 & 0 & 0 \\
\hline 0 & 0 & 0 & 0 & 1 & 0 & 1 & 0 & -1 & 0 & 0 & 0 & 0 \\
\hline 0 & 0 & 0 & 0 & 0 & 1 & 0 & 1 & 0 & -1 & 0 & 0 & 0 \\
\hline 0 & 0 & 0 & 0 & $-r_{3} s_{3}$ & $r_{3} c_{3}$ & $-r_{3} s_{3}$ & $r_{3} c_{3}$ & $\left(r_{3}-l_{3}^{\prime}\right) s_{3}$ & $\left(l_{3}^{\prime}-r_{3}\right) c_{3}$ & 0 & 0 & 0 \\
\hline 0 & 0 & 0 & 0 & 0 & 0 & 0 & 0 & 1 & 0 & -1 & 0 & 0 \\
\hline 0 & 0 & 0 & 0 & 0 & 0 & 0 & 0 & 0 & 1 & 0 & -1 & 0 \\
\hline 0 & 0 & 0 & 0 & 0 & 0 & 0 & 0 & $-r_{4} s_{4}$ & $r_{4} c_{4}$ & $\left(r_{4}-l_{4}^{\prime}\right)$ & $\left.-r_{4}\right)$ & 0 \\
\hline
\end{tabular}

is the matrix of force transformer, $s_{i}=\sin \theta_{i}, c_{i}=\cos \theta_{i}$.

$H_{14 \times 1}=\left[F_{A x^{\prime}}, F_{A z^{\prime}}, F_{B x^{\prime}}, F_{B z^{\prime}}, F_{c x^{\prime}}, F_{c z^{\prime}}, T_{1} \cos \beta, T_{1} \sin \beta\right.$, $\left.F_{D x^{\prime}}, F_{D z^{\prime}}, F_{E x^{\prime}}, F_{E z^{\prime}}, \tau_{1}, \tau_{4}\right]^{T}$ is the vector of resultant and active force.

$B_{12 \times 1}=\left[m_{1} \ddot{x}_{1}^{\prime}, m_{1} g+m_{1} \ddot{z}_{1}^{\prime}, J_{1} \ddot{\theta}_{1}, m_{2} \ddot{x}_{2}^{\prime}, m_{2} g+m_{2} \ddot{z}_{2}^{\prime}\right.$, $\left.J_{2} \ddot{\theta}_{2}, m_{3} \ddot{x}_{3}^{\prime}, m_{3} g+m_{3} \ddot{z}_{3}^{\prime}, J_{3} \ddot{\theta}_{3}, m_{4} \ddot{x}_{4}^{\prime}, m_{4} g+m_{4} \ddot{z}_{4}^{\prime}, J_{4} \ddot{\theta}_{4}\right]^{T}$ is the vector of inertia force.

Taking into account (8) and (14), the dynamic model of the HDCPM is derived from the dynamic equations of the CPM and four groups of the HDPM as follows:

$$
\begin{gathered}
N_{3 \times 4} T_{4 \times 1}=M_{3 \times 1}, \\
A_{12 \times 14}^{i} H_{14 \times 1}^{i}=B_{12 \times 1}^{i} \quad(i=1,2,3,4) .
\end{gathered}
$$

\section{Workspace Analysis}

The workspace of the HDCPM is characterized as the set of points where the end-effector can be positioned while all cables are in tension $\left(T_{i}>0\right)$. At each point within the possible workspace, (8) describing the force in each cable is used to see if tension is obtainable. However, it is not sufficient to obtain the actual workspace of the HDCPM if it only depends on the tension condition. Accordingly, when analyzing the workspace of the HDCPM, one should also consider the following constraints such as the workspace of the HDPM and the motion range of the end-effector.

(1) The workspace of the HDPM. According to (6), the set of points $(x, y, z)$ where the end-effector can be positioned are influenced by cable length $l_{i}$. Cable length $l_{i}$ is calculated by the coordinates joint $C\left(x_{C}^{\prime}, z_{C}^{\prime}\right)$ from (4). Thus, the workspace of the joint $C$ in $x^{\prime}-z^{\prime}$ plane is one of the constraints of the workspace of the HDCPM, and it can be expressed as (only the first group of the HDPM)

$$
\begin{aligned}
& \left|l_{1}^{\prime}-l_{2}^{\prime}\right|<L_{A C}<l_{1}^{\prime}+l_{2}^{\prime}, \\
& \left|l_{3}^{\prime}-l_{4}^{\prime}\right|<L_{C E}<l_{3}^{\prime}+l_{4}^{\prime},
\end{aligned}
$$

where $L_{A C}=\sqrt{x_{C}^{\prime 2}+z_{C}^{\prime 2}}, L_{C E}=\sqrt{\left(x_{C}^{\prime}-l_{5}^{\prime}\right)^{2}+z_{C}^{\prime 2}}$.
(2) The motion range of the end-effector for the HDCPM can be written in the following form:

$$
\begin{gathered}
0<x=\frac{l_{1}^{2}-l_{2}^{2}+a^{2}}{2 a}<a, \\
0<y=\frac{l_{1}^{2}-l_{4}^{2}+b^{2}}{2 b}<b, \\
0<z=h-\sqrt{l_{1}^{2}-x^{2}-y^{2}}<h .
\end{gathered}
$$

A general numerical workspace generation approach is employed here. The possible motion range of joint $C$ in the HDPM is first discretized into a number of points. According to (17), a series of the cable length $l_{i}$ can be calculated. Then, from (18), various combinations of $l_{i}$ can calculate the end-effector coordinates $(x, y, z)$. Thus, a series of points in Cartesian coordinate system are determined. At the end, the points are checked and those satisfying the tension condition $\left(T_{i}>0\right)$ will generate the workspace. The flowchart of generating the workspace of the HDCPM is shown in Algorithm 1.

\section{Results and Discussions}

Simulation studies were performed with the software named MATLAB 2010. A three-dimensional simulation model of the HDCPM has been established, where the four groups of HDPMs have the same structure parameters and symmetry in the three-dimensional space. The parameters of the HDCPM are listed in Table 1. All computations were carried out on a computer with a 2.99-GHz CPU and 2.00 GB memory; the circle trajectory took about $9.67 \mathrm{~s}$, while the line segment trajectory requires only $2.93 \mathrm{~s}$.

5.1. Dynamic Simulation. This section presents two motion cases of the end-effector for dynamic simulation.

Case 1 (circle trajectory). The equation of the circle with a radius of $0.25 \mathrm{~m}$ parallel to the $X-Y$ plane in global coordinate 


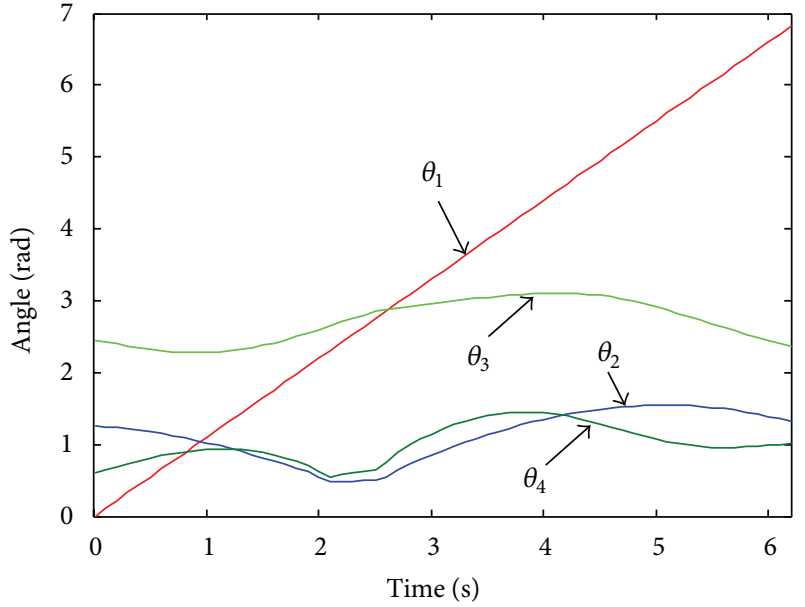

(a)

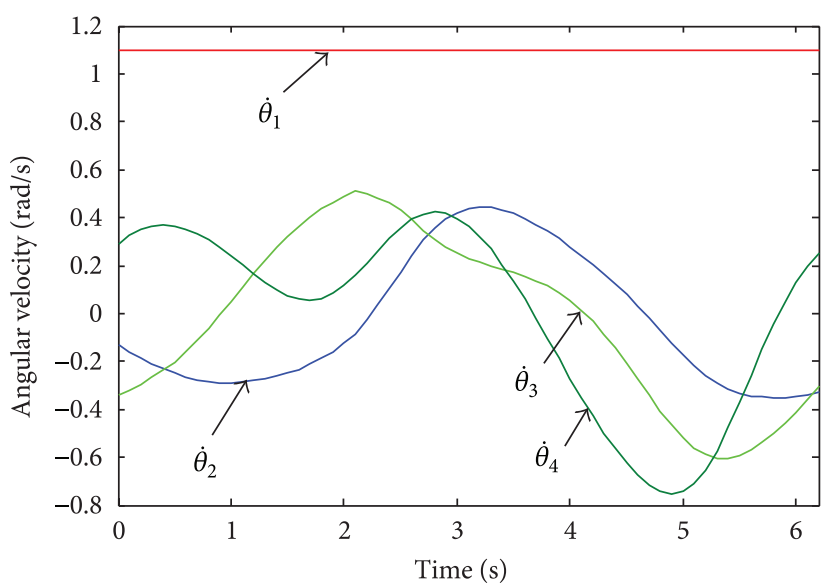

(b)

FIGURE 14: Curves of the links motion parameters of the HDPM as $\omega=1.1 \mathrm{rad} / \mathrm{s}$ : (a) angle and (b) angular velocity.

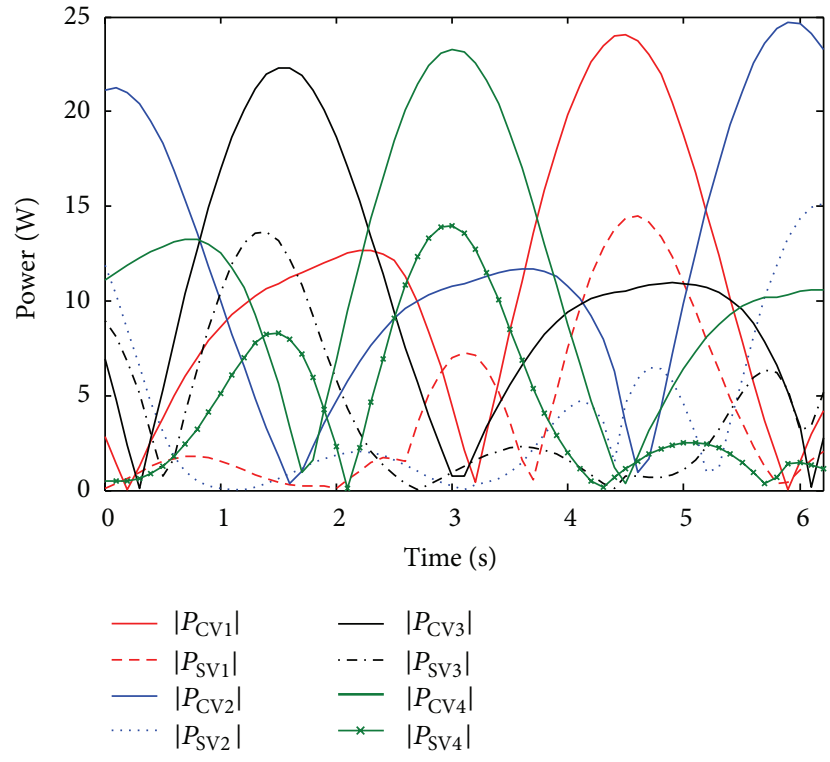

FIGURE 15: Motor absolute value power curves of the four groups of HDPMs of the HDCPM as $\omega=1.1 \mathrm{rad} / \mathrm{s}$.

system is

$$
\begin{gathered}
x=0.25 \cos t+0.5, \\
y=0.25 \sin t+0.5, \\
z=0.5,
\end{gathered}
$$

where $0 \leq t \leq 6.2(\mathrm{~s})$. The end-effector moves along the trajectory with the constant velocity $v=0.25 \mathrm{~m} / \mathrm{s}$ as shown in Figure 5.

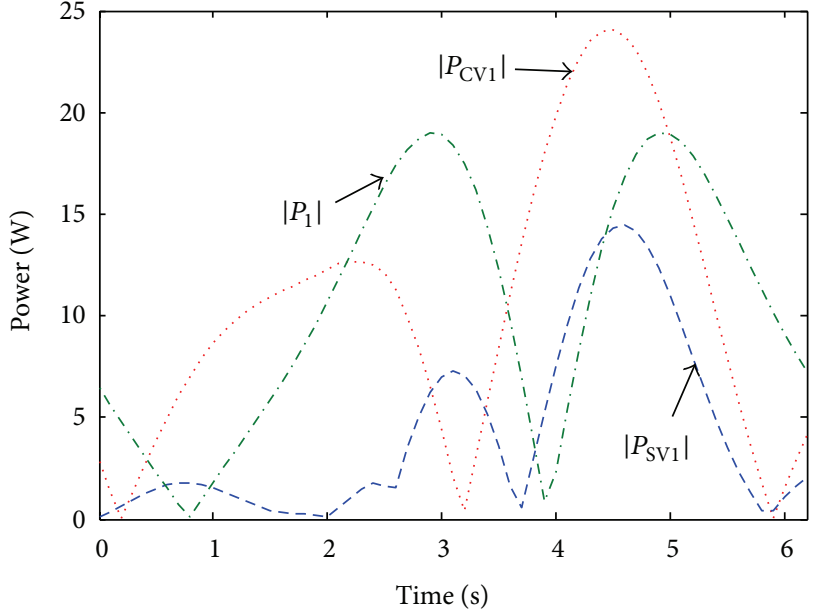

FIGURE 16: Comparison of absolute value power curves between the $\mathrm{HDCPM}$ as $\omega=1.1 \mathrm{rad} / \mathrm{s}$ and conventional CPM.

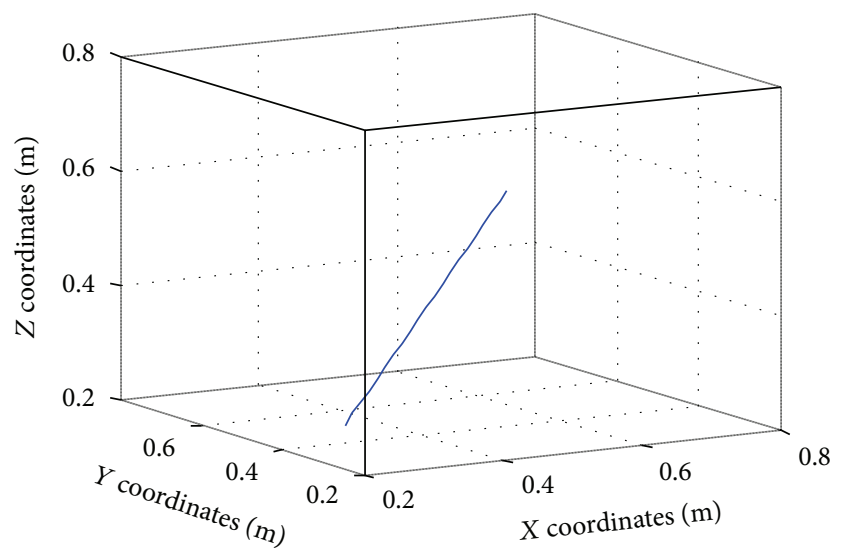

FIGURE 17: Following trajectory of the line segment motion. 


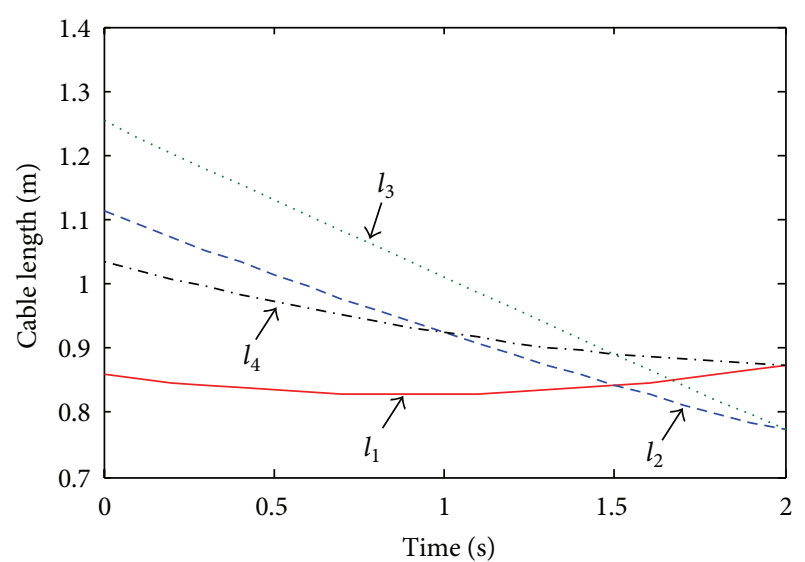

Figure 18: Curves of cable length for Case 2.

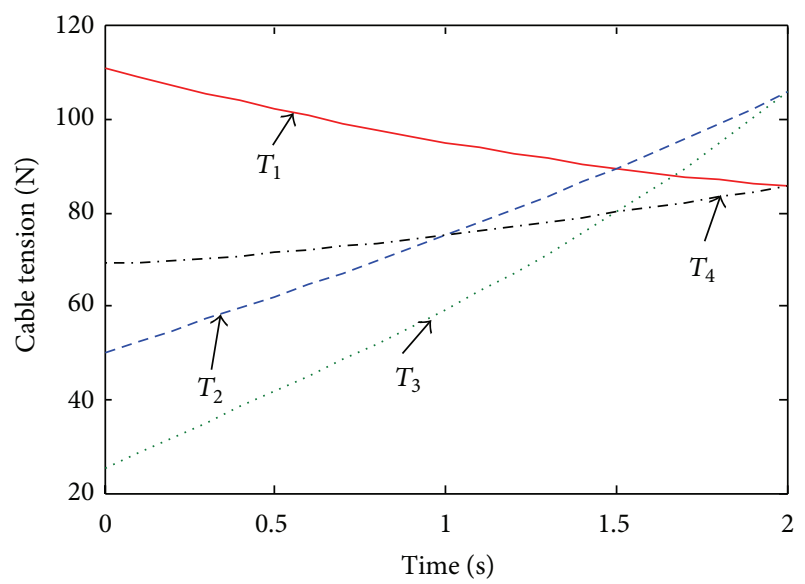

FIgURE 19: Curves of cable tension for Case 2.

Figure 6 shows cable motion in the process of simulation. The cable lengths $l_{i}$ are generated by (5) and vary symmetrically. Figure 7 shows the curves of cable tension exerted on the end-effector related to the circle trajectory. Since the endeffector moves on a horizontal circle trajectory, the changes of cable tensions in Figure 7 are reasonable and the transition is smooth.

The kinematic parameters of the HDPM can be obtained by the inverse kinematics of the HDCPM, for given endeffector position. The angle of joint $A$ is driven by a CV motor and assumed to be

$$
\theta_{1}=\omega t
$$

where $\omega=1 \mathrm{rad} / \mathrm{s}$. According to Figure 3(b) the following geometrical relation is verified:

$$
\begin{aligned}
\left(l_{5}^{\prime}+\right. & \left.p-l_{1}^{\prime} \cos \theta_{1}-l_{2}^{\prime} \cos \theta_{2}\right)^{2}+\left(h-l_{1}^{\prime} \sin \theta_{1}-l_{2}^{\prime} \sin \theta_{2}\right)^{2} \\
= & \left(s-l_{1}\right)^{2},
\end{aligned}
$$

TABLE 1: Parameters of the HDCPM.

\begin{tabular}{lc}
\hline System parameters & Value \\
\hline Mass of the end-effector $m$ & $20 \mathrm{~kg}$ \\
Acceleration due to gravity $g$ & $9.81 \mathrm{~m} / \mathrm{s}^{2}$ \\
Height of the cable tower rack $h$ & $1 \mathrm{~m}$ \\
Side length of four towers & $1 \mathrm{~m}$ \\
distributed in rectangle $a$ & \\
Side length of four towers & $1 \mathrm{~m}$ \\
distributed in rectangle $b$ & $2.3 \mathrm{~m}$ \\
Length of the cable $s$ & $1 \mathrm{~m}$ \\
Length of the $E O p$ & $0.2 \mathrm{~m}$ \\
Length of the Link $A B l_{1}^{\prime}$ & $0.5 \mathrm{~m}$ \\
Length of the Link $B C l_{2}^{\prime}$ & $0.5 \mathrm{~m}$ \\
Length of the Link $C D l_{3}^{\prime}$ & $0.28 \mathrm{~m}$ \\
Length of the Link $E D l_{4}^{\prime}$ & $0.51 \mathrm{~m}$ \\
Length of the Link $A E l_{5}^{\prime}$ & $7.85 \times 10^{-3} \mathrm{~kg} / \mathrm{m}^{3}$ \\
Density of the Links & $2 \times 10^{-4} \mathrm{~m}^{2}$ \\
Cross-sectional area of the Links
\end{tabular}

where $l_{1}$ is known from (5). Combining (20), and (21), $\theta_{2}$ can be calculated as

$$
\theta_{2}=2 \arctan \left(\frac{E_{1}-\sqrt{F_{1}^{2}+E_{1}^{2}-G_{1}^{2}}}{F_{1}-G_{1}}\right),
$$

where $E_{1}=2 l_{2}^{\prime}\left(l_{2}^{\prime} \sin \theta_{1}-h\right), F_{1}=2 l_{2}^{\prime}\left(l_{1} \cos \theta_{1}-l_{5}^{\prime}-p\right), G_{1}=$ $\left(l_{5}^{\prime}+p\right)^{2}-2 l_{1}^{\prime}\left(h \sin \theta_{1}+\left(l_{5}^{\prime}+p\right) \cos \theta_{1}\right)+l_{1}^{\prime 2}+l_{2}^{\prime 2}+h^{2}-\left(s-l_{1}\right)^{2}$.

From (3), (20) and (22), the coordinates of joint $C\left(x_{C}^{\prime}, z_{C}^{\prime}\right)$ can be determined as

$$
\begin{aligned}
& x_{C}^{\prime}=l_{1}^{\prime} \cos \theta_{1}+l_{2}^{\prime} \cos \theta_{2}, \\
& z_{C}^{\prime}=l_{1}^{\prime} \sin \theta_{1}+l_{2}^{\prime} \sin \theta_{2} .
\end{aligned}
$$

According to (3), the coordinates of joint $C\left(x_{C}^{\prime}, z_{C}^{\prime}\right)$ can be rewritten as

$$
\begin{gathered}
x_{C}^{\prime}=l_{5}^{\prime}+l_{3}^{\prime} \cos \theta_{3}+l_{4}^{\prime} \cos \theta_{4}, \\
z_{C}^{\prime}=l_{3}^{\prime} \sin \theta_{3}+l_{4}^{\prime} \sin \theta_{4} .
\end{gathered}
$$

Solving (24), one gets

$$
\begin{gathered}
\theta_{4}=2 \arctan \left(\frac{E_{2}-\sqrt{F_{2}^{2}+E_{2}^{2}-G_{2}^{2}}}{F_{2}-G_{2}}\right), \\
\theta_{3}=\arcsin \left(\frac{z_{C}^{\prime}-l_{4}^{\prime} \sin \theta_{4}}{l_{3}^{\prime}}\right),
\end{gathered}
$$

where $E_{2}=-2 z_{C}^{\prime} l_{4}^{\prime}, F_{2}=2 l_{4}^{\prime} l_{5}^{\prime}-2 x_{C}^{\prime} l_{4}^{\prime}$ and $G_{2}=\left(x_{C}^{\prime}-l_{5}^{\prime}\right)^{2}+$ $z_{C}^{\prime 2}+l_{4}^{\prime 2}-l_{3}^{\prime 2}$.

According to the inverse kinematics analysis of the HDCPM above, one can calculate the angular variables for 


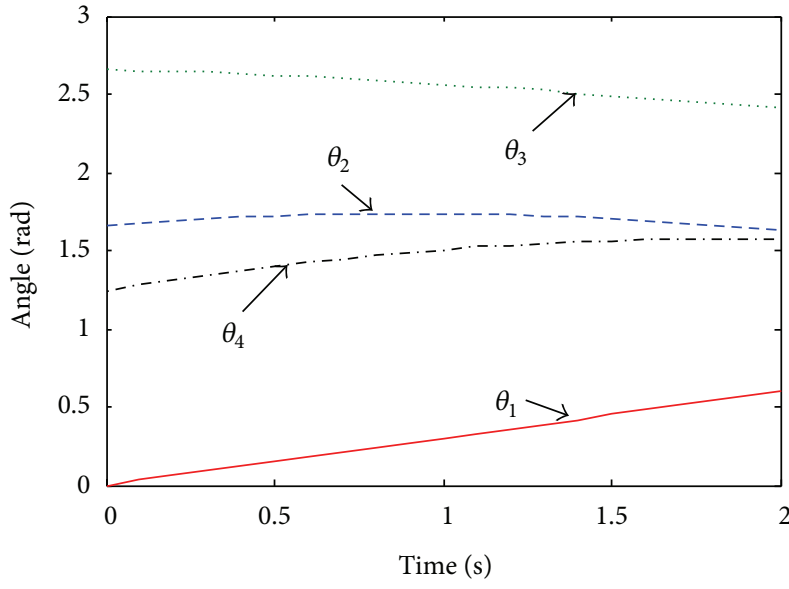

(a)

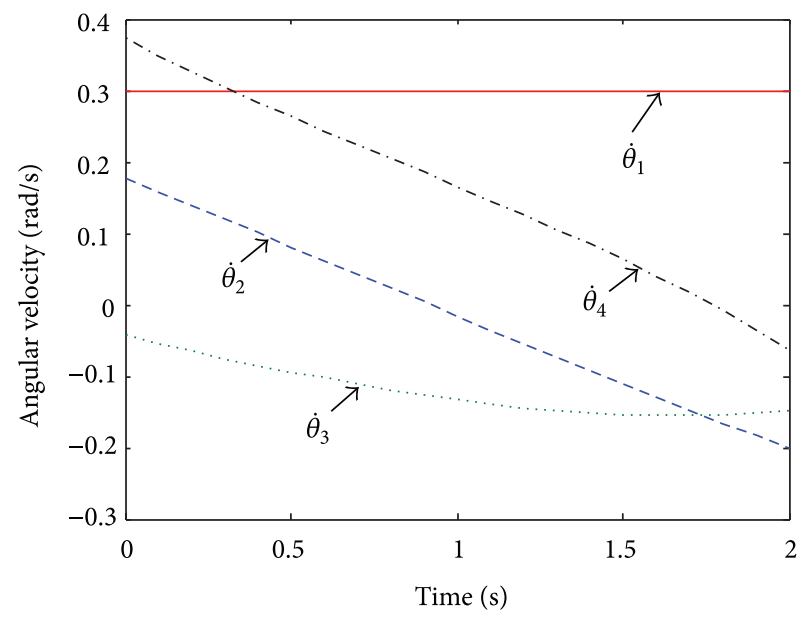

(b)

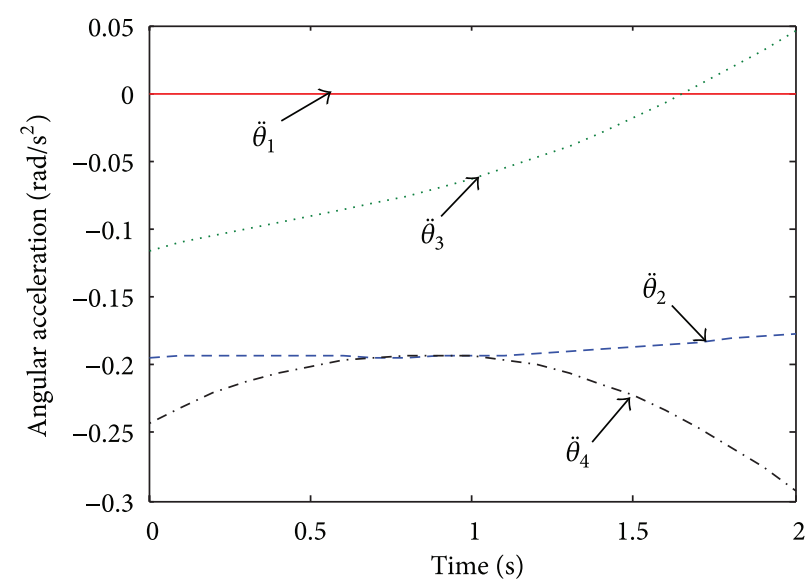

(c)

FIgURE 20: Curves of the links motion parameters of the HDPM for Case 2: (a) angle, (b) angular velocity and (c) angular acceleration.

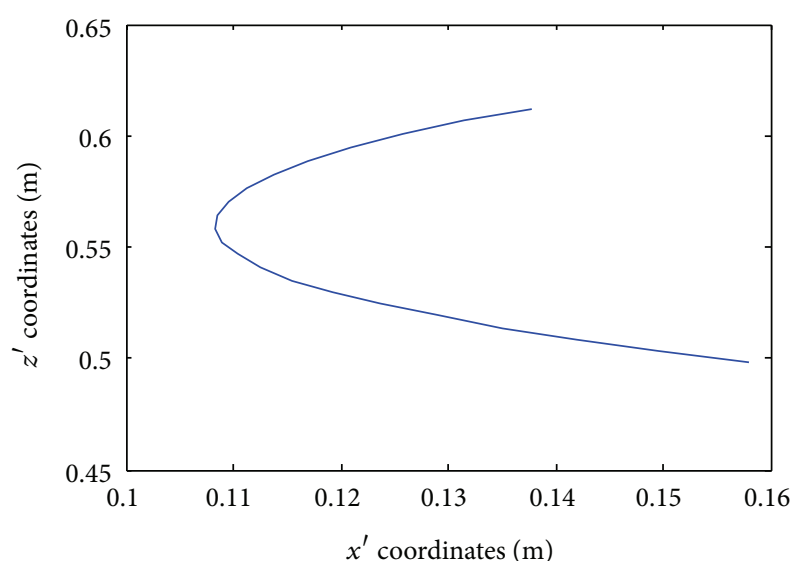

FIGURE 21: Running trajectory of the joint $C$ in $x^{\prime}-z^{\prime}$ plane for Case 2 .

all links of the HDPM as the end-effector moves along the circle. Figure 8 shows the angle curves of the links of the
HDPM. It can be noted that the angular variable law for link $A B$ is a line, while for the other links are curves. This is in line with the motion law of the HDPM. Moreover, angular curves of all the links maintain good continuity, and the numerical values are reasonable. The angular velocity curves and angular acceleration curves of links can be generated by taking the first and second time derivatives of the angular change $\left(d \theta_{i} / d t, d^{2} \theta_{i} / d t^{2}\right)$. The coordinates of the joint $C$ in local $x^{\prime}-z^{\prime}$ plane can be computed by (23). The running trajectory of joint $C$ is depicted in Figure 9.

Figure 10 depicts the resulting force curves of the joints of the HDPM. From it one can see that the resultant force curves of the joints are smooth, which is consistent with the desired motion law.

If the cables are driven only by coils and servomotors, which is a classical approach for the conventional CPM, the range and speed of obtainable output motions may be limited by certain servomotor power capacity and high cost. This problem can be solved by using hybrid-driven machines instead of coils and servomotors. For the hybrid-driven machines, the $\mathrm{CV}$ motor provides the majority of power 


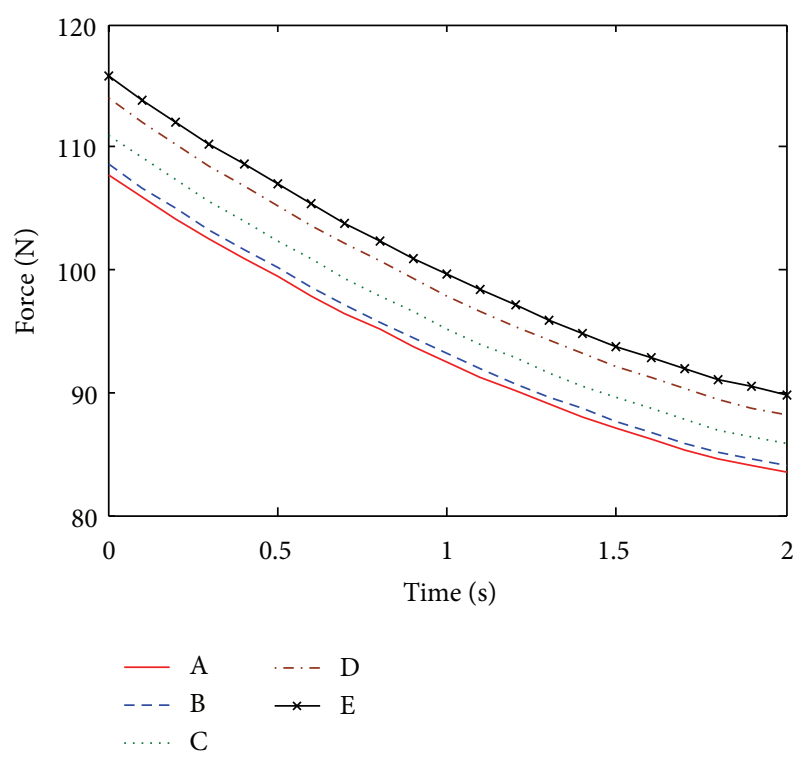

FIGURE 22: Resultant force curves of the joints of the HDPM for Case 2 .

supply to drive the mechanism's motion. The servomotor is real-time controllable and offline programmable [20]. Therefore, the hybrid-driven mechanisms take the advantage of the complementary characteristics of two types of motors to generate programmable output motions with high power capacities in low cost.

The CV motor power and servomotor power of the HDPM can be calculated by

$$
\begin{aligned}
& P_{\mathrm{CV} 1}=\tau_{1} \times \dot{\theta}_{1}, \\
& P_{\mathrm{SV} 1}=\tau_{4} \times \dot{\theta}_{4},
\end{aligned}
$$

where $P_{\mathrm{CV} 1}$ is the $\mathrm{CV}$ motor power of the first group of the HDPM and $P_{\text {SV1 }}$ is the servomotor power of the first group of the HDPM. Similarly, the other motor power, $P_{\mathrm{CV} 2}$, $P_{\mathrm{SV} 2}, P_{\mathrm{CV} 3}, P_{\mathrm{SV} 3}, P_{\mathrm{CV} 4}, P_{\mathrm{SV} 4}$, of the HDPM can be derived, respectively.

Figure 11 shows motor absolute value power curves of four groups of the HDPMs of the HDCPM. From Figure 11, for each of the HDPM, the range of the CV motor power $\left(P_{\mathrm{CV} i}\right)$ is larger than that of the servomotor power $\left(P_{\mathrm{SV} i}\right)$. It is illustrated also that the $\mathrm{CV}$ motors undertake the majority of power for the HDCPM system.

As the motion circular trajectory of the end-effector is the same, and as the structural parameters of the classical approach for the conventional CPM are the same (cables are driven only by coils and servomotors), the servomotor power of the conventional CPM can be obtained by

$$
P_{i}=T_{i} \times \dot{l}_{i} \quad(i=1,2,3,4),
$$

where $P_{i}$ is the $i$ th servomotor power, $T_{i}$ represents the $i$ th cable tension, $\dot{l}_{i}$ denotes the $i$ th cable velocity.

Figure 12 shows the servomotor absolute value power curves of the classical approach for the conventional CPM.
For comparison, absolute value power curves of the first group of HDPM of the HDCPM and the first servomotor of the conventional CPM are given in Figure 13. As it is seen, large range of servomotor power $\left|P_{1}\right|$ of the conventional CPM may be decreased by using the HDPM.

In order to investigate the power property of the HDCPM further, the following simulation is performed as the $\mathrm{CV}$ motors of the four groups of the HDPMs of the HDCPM run with $1.1 \mathrm{rad} / \mathrm{s}(\omega=1.1 \mathrm{rad} / \mathrm{s})$. Figure 14 displays curves of the link angle and velocity of the HDPM. Angular curves of all links maintain good continuity, and the numerical values are reasonable. Figure 15 shows absolute value power curves of four groups of the HDPMs of the HDCPM. Figure 16 illustrates absolute value power curves of the first group of HDPM of the HDCPM and the first servomotor of the conventional CPM. From the simulations, we can see that the CV motors provide the majority of power supply and the servomotors mainly act as a motion regulation device as depicted in Figures 11, 13, 15, and 16.

Case 2 (line segment trajectory). The equation of the line in global coordinate system is

$$
\begin{aligned}
& x=\frac{t}{6}+0.25 \\
& y=\frac{t}{12}+\frac{1}{3}, \\
& z=\frac{t}{6}+0.25
\end{aligned}
$$

where $0<t<2$ (s). From (28), it can be noted that the center of gravity of the end-effector moves the geometric path that is line segment with the constant velocity $v=0.25 \mathrm{~m} / \mathrm{s}$, the initial point is $(0.25,1 / 3,0.25)(\mathrm{m})$. The trajectory tracking of the end-effector related to Case 2 is shown in Figure 17. From this figure the formulation tracks the planned trajectory relatively well.

Figure 18 shows the changes of cables lengths. As can be seen, the variations of the curves of cables lengths are in accordance with the reference path (28) of the end-effector.

Figure 19 depicts the curves of cable tensions for Case 2. One can see that the curves are smooth, which is the ideal data for the HDCPM system which operates steadily.

The angular variables of links of the HDPM for Case 2 can be obtained by the inverse kinematics analysis of the HDCPM. The angle curves of the HDPM links are shown in Figure 20(a). The corresponding angular velocity and angular acceleration are shown in Figures 20(b) and 20(c). Figure 21 shows the running trajectory of the joint $C$ in local $x^{\prime} o^{\prime} z^{\prime}$ plane.

The resulting force curves of the joints of the HDPM for Case 2 can be solved by the dynamics modeling of the HDCPM in Section 3, as shown in Figure 22. It can be noted that the resulting force curves of the joints have the same trend and change smoothly. Comparison of absolute value power curves of the HDPMs in the HDCPM and the servomotors of the conventional CPM for Case 2 are shown in Figure 23. Referring to Figure 23, it can be seen that the 

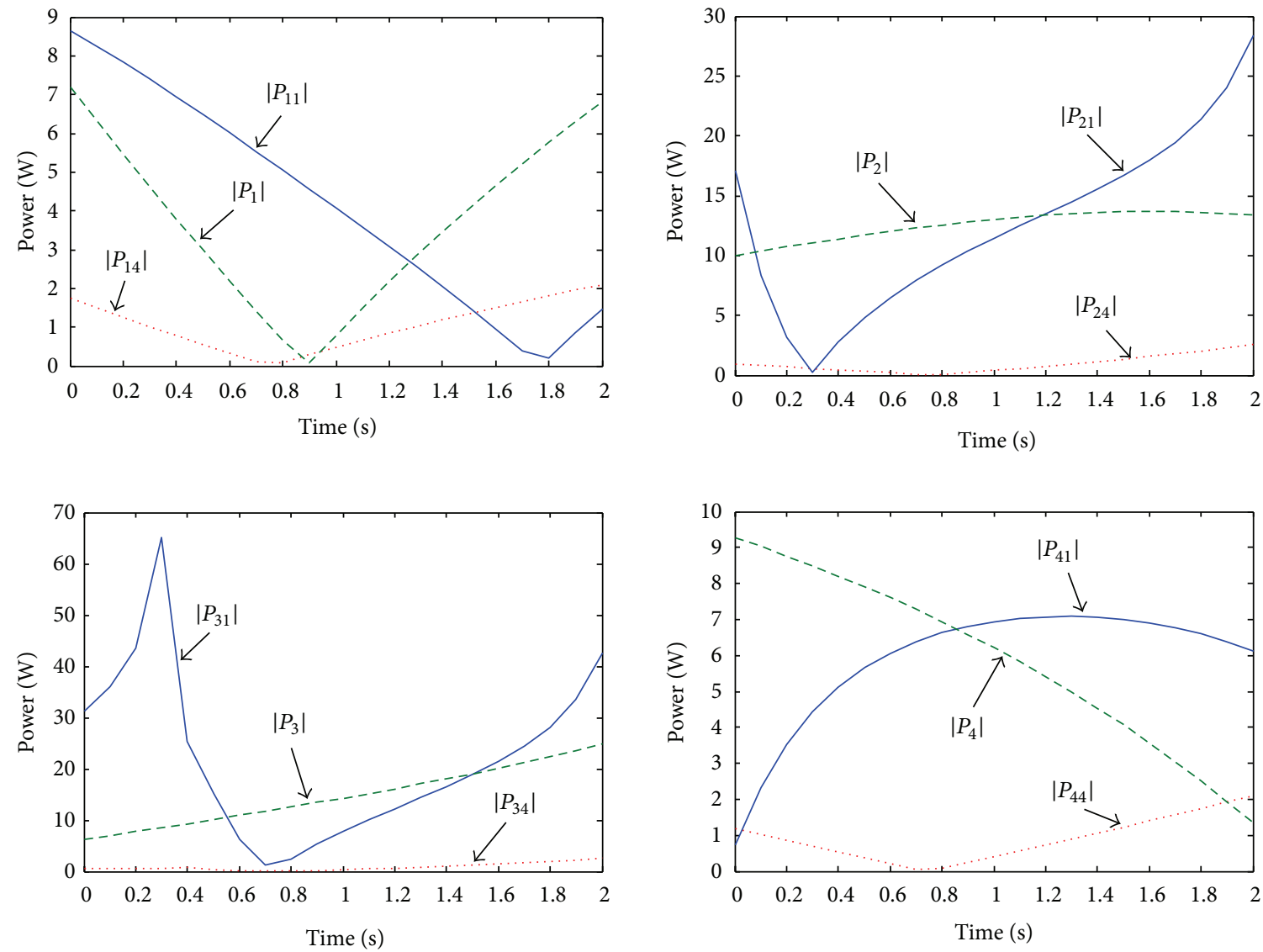

FIGURE 23: Comparison of absolute value power curves between the HDCPM and conventional CPM for Case 2.

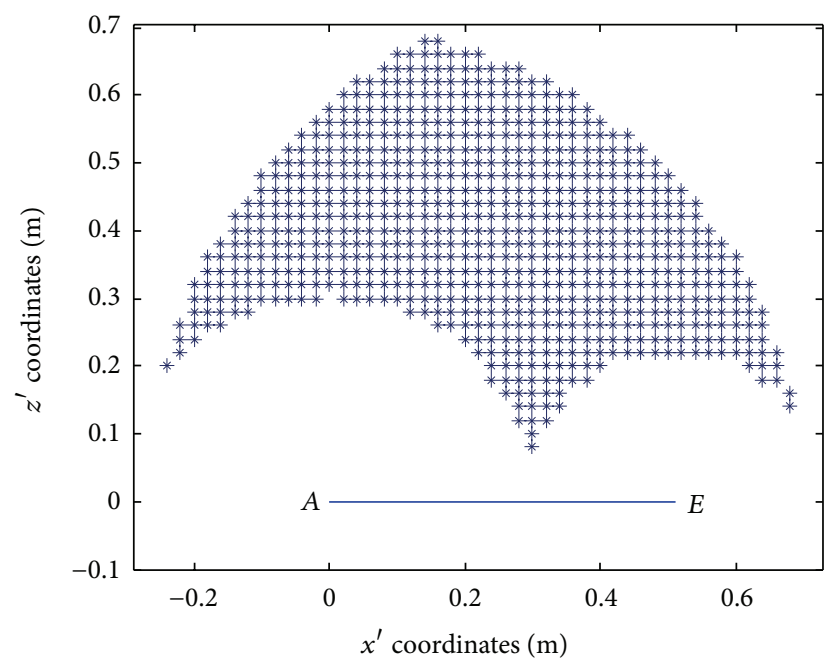

Figure 24: Workspace of joint $C$ in $x^{\prime}-z^{\prime}$ plane.

$\mathrm{CV}$ motors provide the majority of power supply and the servomotors mainly act as a motion regulation device.

From the results and discussions, illustrative simulation studies highlight its performances, which lay a foundation for the further research on optimization and real-time control.
5.2. Workspace Simulation. The workspace of joint $C$ is determined by geometric constraints (17) given in Figure 24. The simulation results of the reachable 3D workspace for the HDCPM and the projections of workspace onto the $X-Y$ plane and $X-Z$ plane are shown in Figure 25. The whole workspace is approximately an upside-down cone. It should be noted that most of the workspace volume is concentrated in the upper part of the tower cube, which is important for trajectory planning of the end-effector.

\section{Concluding Remarks}

This paper describes the theoretical investigations of a class of the completely restrained hybrid-driven based cable parallel manipulators (HDCPMs) with three translational motions. The HDCPM combines the advantages of four groups of HDPMs and a 4-cable parallel manipulator in a way to provide a solution for moving heavy objects with high efficiency and high performance. Detailed design and implementation of a virtual prototype model of the HDCPM is presented as well. Dynamic modeling of the full system including both the mechanical system and the driving system is addressed. The inverse kinematic and dynamic problems of the HDCPM system are resolved on condition that an operation path of the end-effector has been planned. Then, the main factors, which influence the workspace of the HDCPM such as workspace 


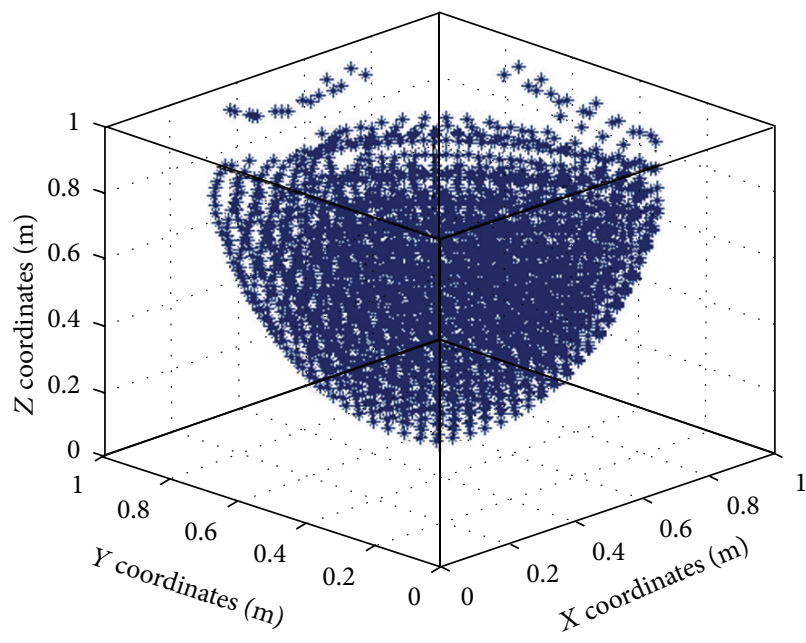

(a)

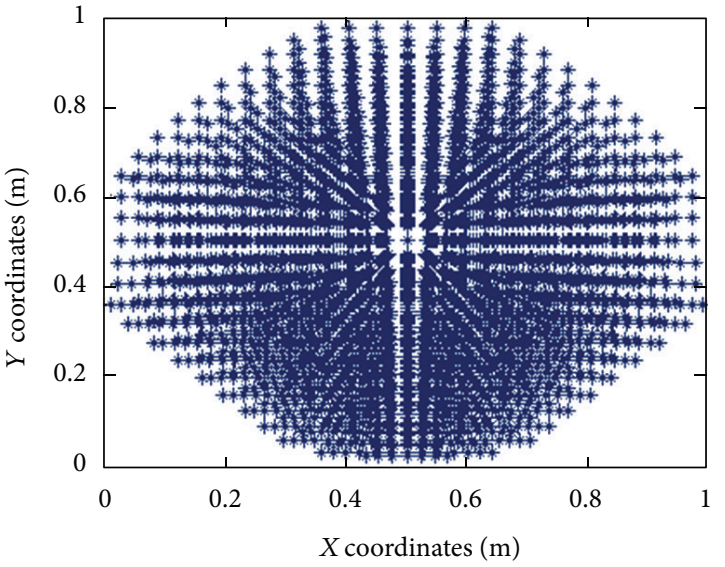

(b)

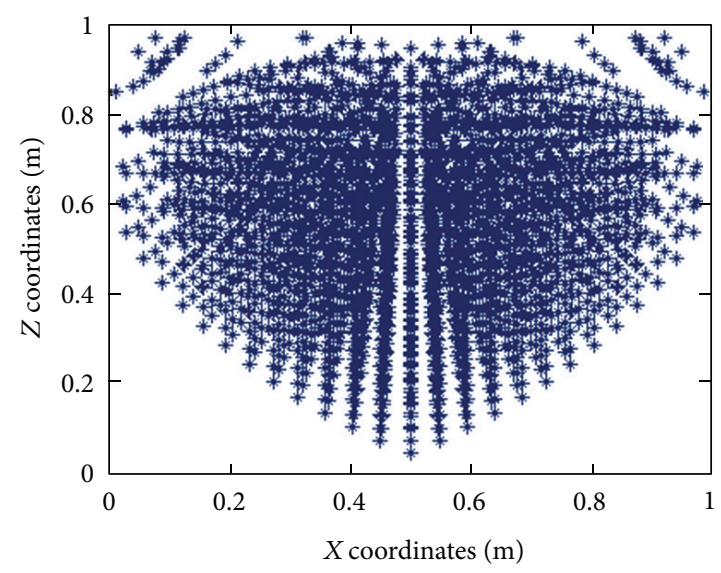

(c)

FIgURE 25: Workspace of the HDCPM (a) and projections of workspace onto $X-Y$ plane (b) and $X-Z$ plane (c).

of the HDPM and the length of cables, are analyzed based on the structure constraints of the HDCPM. Finally, illustrative examples for trajectory tracking and workspace demonstrate that the manipulator design is reasonable and the associated models are correct.

The prototype verification of the designed HDCPM is currently under investigation. Furthermore, more work is in progress to implement control system design, design modification, and optimum synthesis for the HDCPM. In addition, the methodology presented in this work could be used as a guide for the design and analysis of other manipulators.

\section{Acknowledgments}

This work was supported by the National Natural Science Foundation of China (50905179, 51275515), Program for Innovative Research Team in University (IRT1292), the Six Talent Peak Foundation of Jiangsu Province, and the Priority Academic Programme Development of Jiangsu Higher Education Institutions. The authors also appreciate the comments and valuable suggestions of anonymous referees and editors to improve the quality of the paper.

\section{References}

[1] B. Zi, Z. C. Zhu, and J. L. Du, "Analysis and control of the cablesupporting system including actuator dynamics," Control Engineering Practice, vol. 19, no. 5, pp. 491-501, 2011.

[2] W. B. Lim, G. Yang, S. H. Yeo, and S. K. Mustafa, "A generic force-closure analysis algorithm for cable-driven parallel manipulators," Mechanism and Machine Theory, vol. 46, no. 9, pp. 1265-1275, 2011.

[3] S. R. Oh and S. K. Agrawal, "A reference governor-based controller for a cable robot under input constraints," IEEE Transactions on Control Systems Technology, vol. 13, no. 4, pp. 639-645, 2005.

[4] A. Alikhani, S. Behzadipour, A. Alasty, and S. A. S. Vanini, "Design of a large-scale cable-driven robot with translational motion," Robotics and Computer-Integrated Manufacturing, vol. 27, no. 2, pp. 357-366, 2011.

[5] A. D. Dimarogonas, Machine Design: A CAD Approach, John Wiley \& Sons, New York, NY, USA, 2001. 
[6] N. Zrnic, K. Hoffmann, and S. Bosnjak, "A note on the history of handling in ports: from ancient to medieval cranes," in Proceedings of the 12th IFToMM World Congress, Besancon, France, 2007.

[7] T. G. Chondros, "Archimedes life works and machines," Mechanism and Machine Theory, vol. 45, no. 11, pp. 1766-1775, 2010.

[8] B. Y. Duan, Y. Y. Qiu, F. S. Zhang, and B. Zi, "On design and experiment of the feed cable-suspended structure for super antenna," Mechatronics, vol. 19, no. 4, pp. 503-509, 2009.

[9] M. H. Korayem and M. Bamdad, "Dynamic load-carrying capacity of cable-suspended parallel manipulators," International Journal of Advanced Manufacturing Technology, vol. 44, no. 7-8, pp. 829-840, 2009.

[10] S. Lahouar, E. Ottaviano, S. Zeghoul, L. Romdhane, and M. Ceccarelli, "Collision free path-planning for cable-driven parallel robots," Robotics and Autonomous Systems, vol. 57, no. 11, pp. 1083-1093, 2009.

[11] C. M. Gosselin and G. Barrette, "Kinematic analysis of planar parallel mechanisms actuated with cables," in Proceedings of the Symposium on Mechanisms, Machines and Mechatronics, Quebec, Canada, 2001.

[12] E. Ottaviano, M. Ceccarelli, A. Paone, and G. Carbone, "A low-cost easy operation 4-cable driven parallel manipulator," in Proceedings of the IEEE International Conference on Robotics and Automation (ICRA '05), pp. 4008-4013, Barcellona, Spain, April 2005.

[13] T. Heyden and C. Woernle, "Dynamics and flatness-based control of a kinematically undetermined cable suspension manipulator," Multibody System Dynamics, vol. 16, no. 2, pp. 155-177, 2006.

[14] O. Baser and E. I. Konukseven, "Theoretical and experimental determination of capstan drive slip error," Mechanism and Machine Theory, vol. 45, no. 6, pp. 815-827, 2010.

[15] Y. $\mathrm{Li}$ and Q. Xu, "Kinematic analysis and design of a new 3DOF translational parallel manipulator," Journal of Mechanical Design, vol. 128, no. 4, pp. 729-737, 2006.

[16] S. Bouchard and C. M. Gosselin, "Workspace optimization of a very large cable-driven parallel mechanism for a radiotelescope application," in Proceedings of the ASME International Design Engineering Technical Conferences and Computers and Information in Engineering Conference, pp. 963-970, Las Vegas, Nev, USA, September 2007.

[17] R. Yao, X. Tang, J. Wang, and P. Huang, "Dimensional optimization design of the four-cable-driven parallel manipulator in fast," IEEE/ASME Transactions on Mechatronics, vol. 15, no. 6, pp. 932-941, 2010.

[18] K. Kozak, Q. Zhou, and J. Wang, "Static analysis of cabledriven manipulators with non-negligible cable mass," IEEE Transactions on Robotics, vol. 22, no. 3, pp. 425-433, 2006.

[19] P. H. Borgstrom, N. P. Borgstrom, M. J. Stealey et al., "Design and implementation of NIMS3D, a 3-D cabled robot for actuated sensing applications," IEEE Transactions on Robotics, vol. 25, no. 2, pp. 325-339, 2009.

[20] L. Cheng, Y. Lin, Z.-G. Hou, M. Tan, J. Huang, and W. J. Zhang, "Adaptive tracking control of hybrid machines: a ClosedChain Five-Bar Mechanism Case," IEEE/ASME Transactions on Mechatronics, vol. 99, pp. 1-9, 2010.

[21] P. R. Ouyang, Q. Li, W. J. Zhang, and L. S. Guo, "Design, modeling and control of a hybrid machine system," Mechatronics, vol. 14, no. 10, pp. 1197-1217, 2004.
[22] B. Zi, J. B. Cao, and Z. C. Zhu, "Dynamic simulation of hybrid-driven planar five-bar parallel mechanism based on Simmechanics and tracking control," International Journal of Advanced Robotic System, vol. 8, no. 4, pp. 28-33, 2011.

[23] B. Zi, X. Wu, J. Lin, and Z. C. Zhu, "Inverse kinematics and singularity analysis for a 3-DOF hybrid-driven cablesuspended parallel robot," International Journal of Advanced Robotic Systems, vol. 9, no. 133, pp. 1-9, 2012.

[24] C. M. Anupoju, C. Y. Su, and M. Oya, "Adaptive motion tracking control of uncertain nonholonomic mechanical systems including actuators dynamics," IEE Proceedings-Control Theory and Applications, 152, no. 5, pp. 575-580, 2005.

[25] G. Castelli, E. Ottaviano, and M. Ceccarelli, "A fairly general algorithm to evaluate workspace characteristics of serial and parallel manipulators," Mechanics Based Design of Structures and Machines, vol. 36, no. 1, pp. 14-33, 2008.

[26] C. B. Pham, S. H. Yeo, G. Yang, and I. M. Chen, "Workspace analysis of fully restrained cable-driven manipulators," Robotics and Autonomous Systems, vol. 57, no. 9, pp. 901-912, 2009.

[27] O. Altuzarra, J. Aginaga, A. Hernández, and I. Zabalza, "Workspace analysis of positioning discontinuities due to clearances in parallel manipulators," Mechanism and Machine Theory, vol. 46, no. 5, pp. 577-592, 2011.

[28] J. Pusey, A. Fattah, S. Agrawal, and E. Messina, "Design and workspace analysis of a 6-6 cable-suspended parallel robot," Mechanism and Machine Theory, vol. 39, no. 7, pp. 761-778, 2004. 


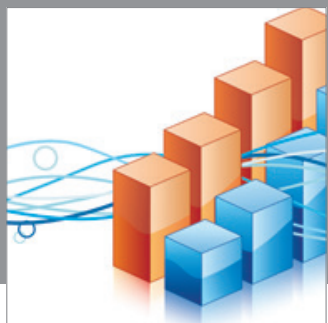

Advances in

Operations Research

mansans

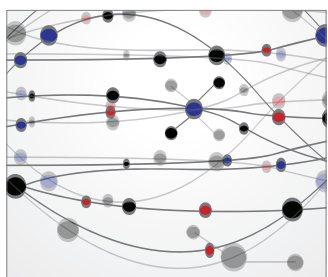

The Scientific World Journal
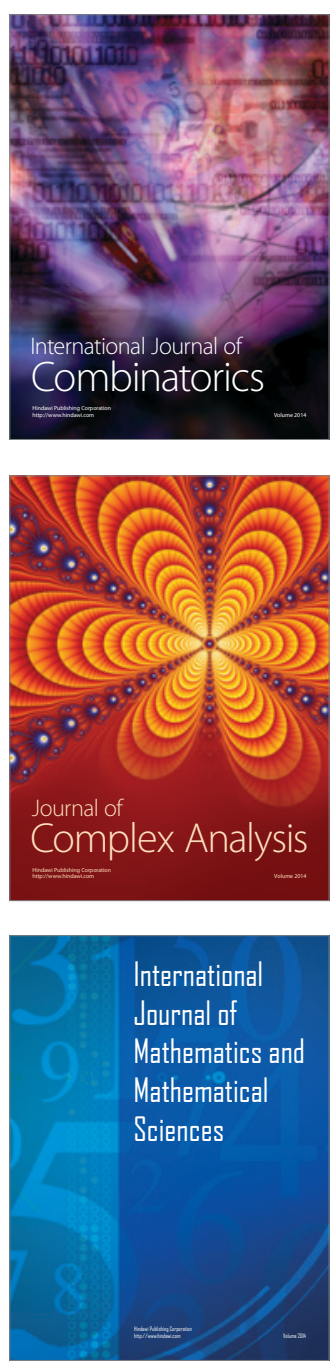
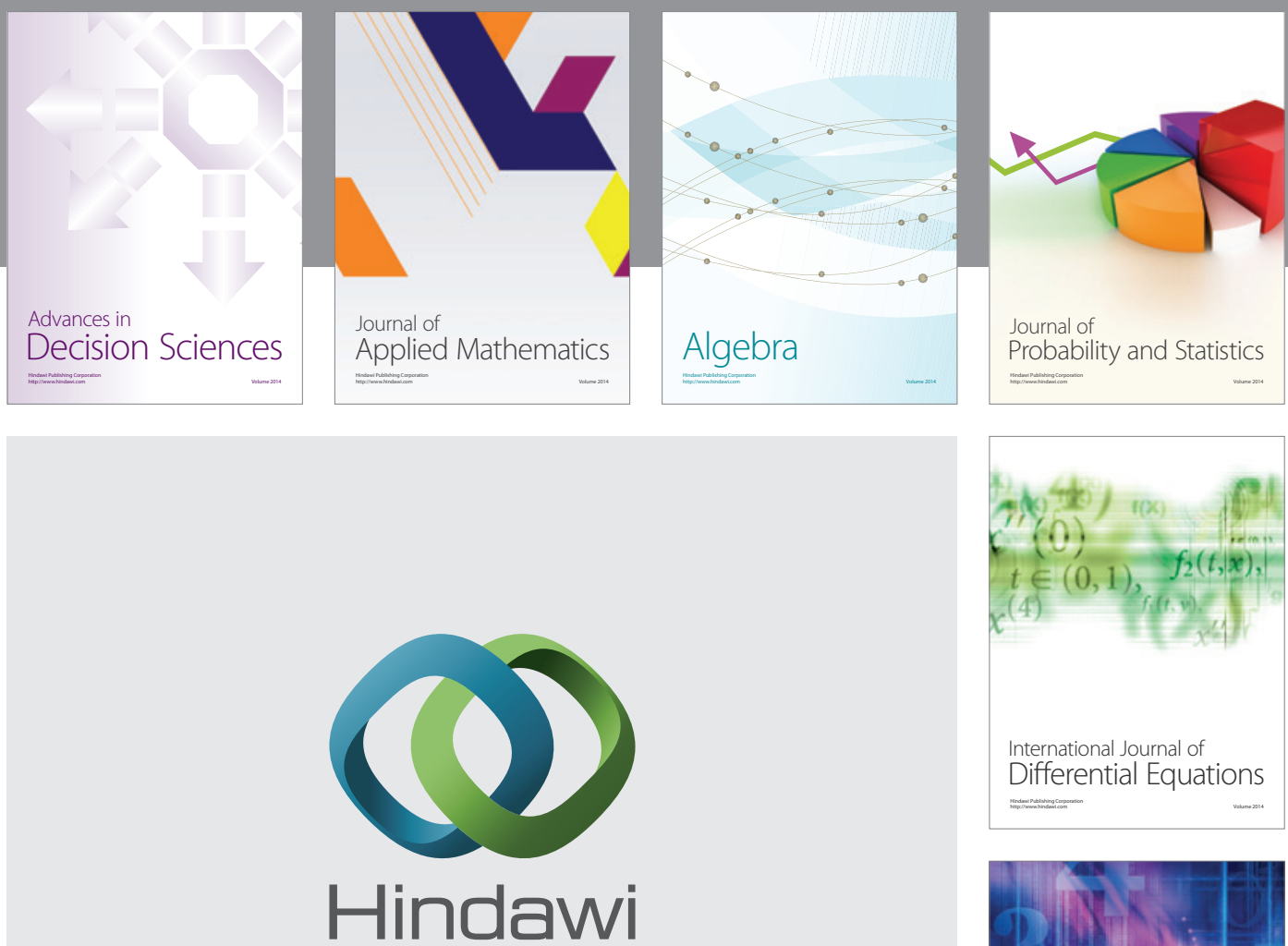

Submit your manuscripts at http://www.hindawi.com
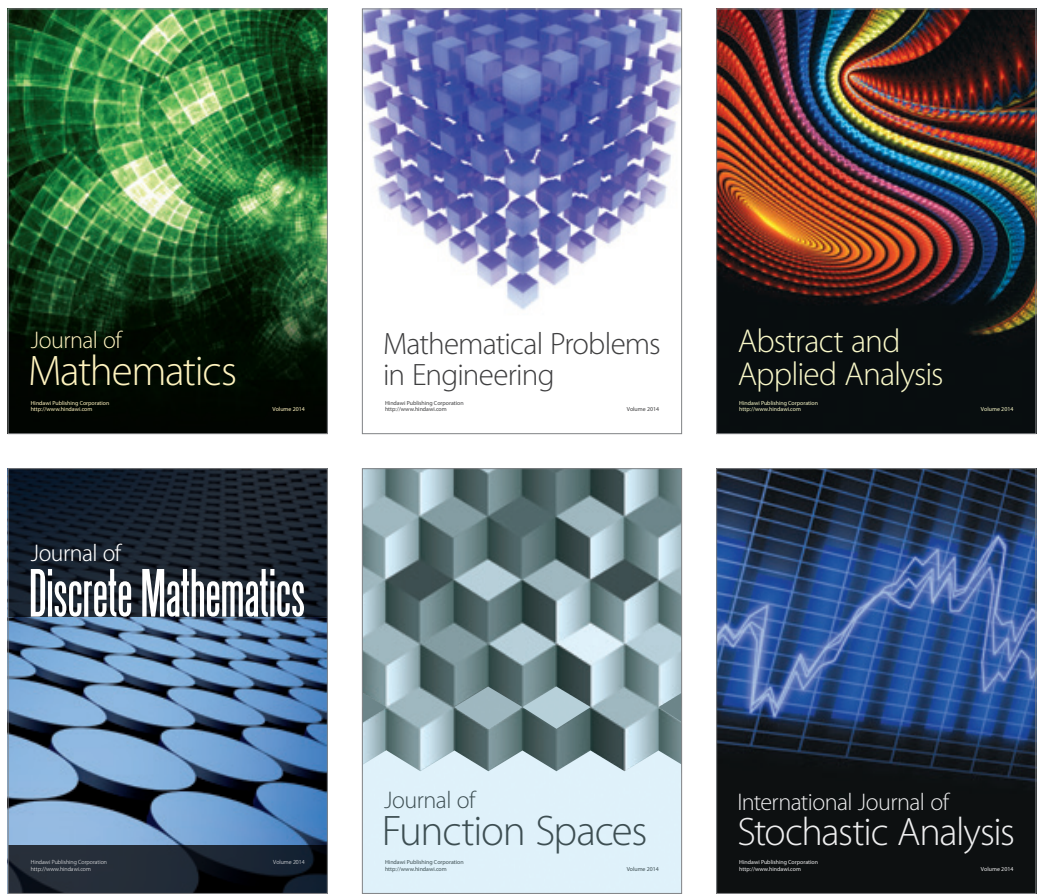

Journal of

Function Spaces

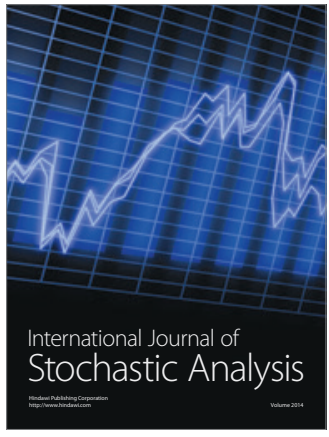

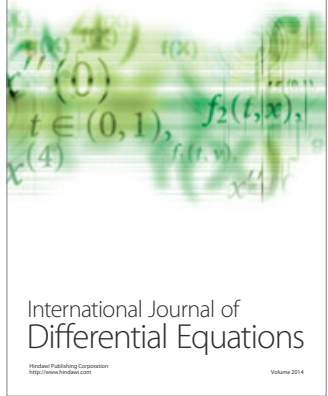
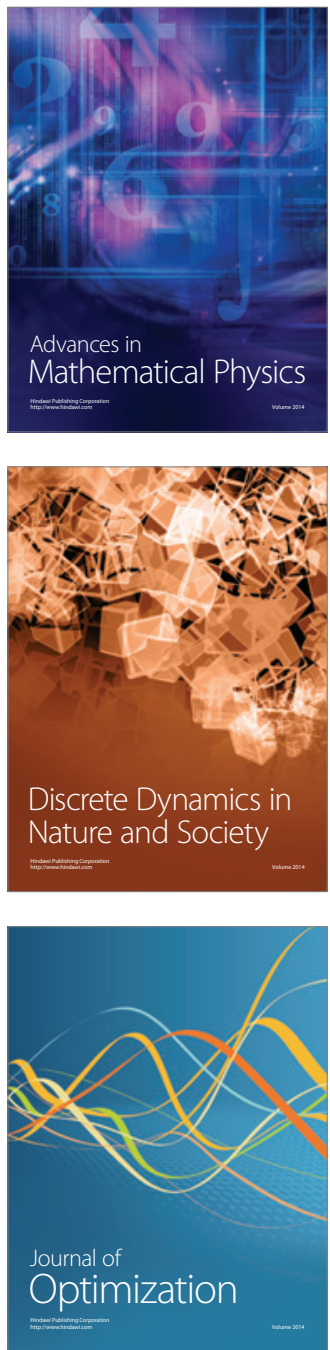